\title{
An Adaptive Noise Removal Approach for Restoration of Digital Images Corrupted by Multimodal Noise
}

\author{
Ovidiu Ghita, Dana E. Ilea and Paul F. Whelan \\ Centre for Image Processing and Analysis \\ Dublin City University, Dublin 9, Ireland
}

\begin{abstract}
Data smoothing algorithms are commonly applied to reduce the level of noise and eliminate the weak textures contained in digital images. Anisotropic diffusion algorithms form a distinct category of noise removal approaches that implement the smoothing process locally in agreement with image features such as edges that are typically determined by applying diverse partial differential equation (PDE) models. While this approach is opportune since it allows the implementation of feature-preserving data smoothing strategies, the inclusion of the PDE models in the formulation of the data smoothing process compromises the performance of the anisotropic diffusion schemes when applied to data corrupted by non-Gaussian and multimodal image noise.

In this paper we first evaluate the positive aspects related to the inclusion of a multi-scale edge detector based on the generalisation of the Di Zenzo operator into the formulation of the anisotropic diffusion process. Then, we introduce a new approach that embeds the vector median filtering into the discrete implementation of the anisotropic diffusion in order to improve the performance of the noise removal algorithm when applied to multimodal noise suppression. To evaluate the performance of the proposed data smoothing strategy, a large number of experiments on various types of digital images corrupted by multimodal noise were conducted.
\end{abstract}

Keywords - Anisotropic diffusion, vector median filtering, feature preservation, multimodal noise, noise removal. 


\section{Introduction}

Noise reduction algorithms are widely accepted as fundamental precursors to early computer vision tasks such as edge detection, data clustering and image segmentation. Over the past few decades numerous approaches have been proposed where the main objective was the implementation of feature preserving noise removal strategies. In the development of noise removal algorithms the prior knowledge about the noise distribution is essential (where the most common are the Gaussian distributed and the impulse noise $[1,2])$ and the main efforts were focused on the development of optimal strategies that addressed the accurate image restoration for one particular noise model. For instance, when dealing with Gaussian distributed noise, the image restoration process can be achieved by applying local averaging operators such as Gaussian filtering [2], but it is useful to note that the noise removal is obtained at the cost of attenuating contextual features such as edges. To circumvent this undesired effect associated with linear smoothing strategies, the noise removal process has been reformulated using non-linear schemes in order to achieve the preservation of meaningful contextual features. A characteristic of many feature preserving non-linear noise removal techniques is that they incorporate the gradient information into the data smoothing process and this leads to a considerable reduction in their performance when applied to data corrupted by non-Gaussian distributed noise (and in a more generic case to images corrupted by multimodal noise, where at least one component of the multimodal noise has a non-Gaussian probability density function). In this paper we focus our attention on noise removal strategies based on anisotropic diffusion [3-11] to improve their performance when dealing with non-Gaussian distributed noise. To achieve this goal we propose the inclusion of the vector median filtering (VMF) [12-16] approach into the formulation of the anisotropic diffusion (AD), where the purpose is the development of an image restoration strategy that outperforms both the $\mathrm{AD}$ and VMF algorithms when applied to data corrupted by multimodal image noise. The main contributions associated with this work are located in the inclusion of a multi-scale edge detector into the formulation of the Perona-Malik (PM) anisotropic diffusion scheme [3] and in the implementation of a new noise removal framework that is able to restore digital images that are corrupted by multimodal noise. 
This paper is organised as follows. In Section 2 the multi-scale anisotropic diffusion algorithm is introduced. Section 3 details the VMF denoising technique and the proposed multi-stage image restoration scheme. Section 4 presents the experimental results, while Section 5 concludes this paper.

\section{Anisotropic Diffusion. Problem Formulation}

The anisotropic diffusion noise removal strategy has been initially proposed by Perona and Malik in [3] where the data smoothing has been formulated based on the principles associated with the heat diffusion process. Using this concept, data smoothing is performed inside the perceptual coherent image regions and suppressed at regions' boundaries. This feature-preserving noise removal approach can be efficiently defined in terms of the derivative of the flux function. If we assume that the image is defined as a discrete signal $I(x, y): \boldsymbol{R}^{\mathrm{m}} \rightarrow \boldsymbol{R}^{\mathrm{n}} \mid(x, y) \in \Gamma, \Gamma \subset \mathbf{R}^{2}$ is the image domain and for a standard color image $m=2$ and $n=$ 3 , then the anisotropic diffusion can be formulated as follows,

$$
\frac{\partial I(x, y, t)}{\partial t}=\operatorname{div}[D(|\nabla I(x, y, t)|) \nabla I(x, y, t)]
$$

where $I(x, y)$ denotes the intensity of the pixel at position $(x, y)$ in the image $I, \nabla I(x, y, t)$ denotes the gradient calculated for the pixel $(x, y)$ at iteration $t, d i v$ is the divergence operator and $D($.$) is the diffusion$ function. In equation $(1), D($.$) is a monotonically decreasing function bounded in the interval (0,1]$ that decays with the increase in the value of the gradient. Typically, the diffusion function is implemented using either exponential or reciprocal forms as illustrated in equation (2),

$$
\begin{gathered}
D(|\nabla I(x, y, t)|)=e^{-\left(\frac{|\nabla I(x, y, t)|}{d}\right)^{2}}, \\
D(|\nabla I(x, y, t)|)=\frac{1}{1+\left(\frac{|\nabla I(x, y, t)|}{d}\right)^{2}}, d>0
\end{gathered}
$$

where $d$ is the diffusion parameter that controls the strength of the filtering process. While the implementation in the discrete domain of the Perona-Malik (PM) anisotropic diffusion shown in equation 
(1) involves an iterative scheme, the standard PM formulation generates substantial errors when the intensity differences between the pixel of interest $(x, y)$ and its neighboring pixels do not approximate a normal distribution. An effective solution to improve the numerical stability of the PM formulation resides in the extraction of the gradient information from the image that is convolved with a Gaussian function $\left(\nabla I(x, y, t) \leftarrow \nabla\left[G_{k} * I(x, y, t)\right]\right.$, where * defines the convolution operator and $k$ is the scale of the Gaussian function). To provide more local (contextual) support for true edges during the filtering process, in this paper the diffusion functions are calculated in the multi-scale sense as illustrated in equation (3).

$$
D(|\nabla(x, y, t)|)=e^{-\left(\frac{\int \in\left[\nabla\left[G_{k} * I(x, y, t)\right] \mid d k\right.}{k \in[0, s]}\right)^{2}},
$$

where $G_{k}$ is the Gaussian function with standard deviation $k, d$ is the diffusion parameter and $s$ denotes the number of scales. As illustrated in equation (1), the stability of the smoothing process is in direct relationship with the accuracy in the calculation of the edge information in the image. In the original formulation proposed by Perona and Malik [3] the gradient operator is calculated using the four-connected neighbourhood differences and this approach proved inefficient when applied to data characterised by weak edges and corrupted by noise [17]. To alleviate these problems, in this paper we propose to extract the edge information using a multi-scale structure tensor and this procedure will be detailed in the next section of the paper. It is useful to mention that other notable improvements to the standard (PM) anisotropic diffusion equation are represented by the forward and backward (FAB) anisotropic diffusion $[7,9,10]$ and the implementation of time-controlled formulations [21] that prevent the suppression of the medium gradients during the iterative noise removal process. We will not discuss these techniques in this paper since the numerical stability of the diffusion equation is beyond the scope of this study. 


\subsection{Multi-scale edge detector}

In the context of two-dimensional images, the structure tensor is a matrix representation of the partial derivatives calculated in two orthogonal directions $[17,18]$ and it has been widely applied to extract lowlevel features in digital images such as edges. To alleviate the sampling problems associated with the structure tensor as described by Di Zenzo [19], in this paper we propose to generalise the standard formulation in the multi-scale sense. Thus, in our implementation the structure tensor is calculated for each pixel at the specified scale $k$ in the image as follows,

$$
M_{k}(x, y)=\sum\left[\nabla f(x, y) \nabla f(x, y)^{T}\right]=\left[\begin{array}{cc}
\sum_{p=1}^{n}\left(\frac{\partial f_{p}}{\partial x}\right)^{2} & \sum_{p=1}^{n}\left(\frac{\partial f_{p}}{\partial x} \frac{\partial f_{p}}{\partial y}\right) \\
\sum_{p=1}^{n}\left(\frac{\partial f_{p}}{\partial x} \frac{\partial f_{p}}{\partial y}\right) & \sum_{p=1}^{n}\left(\frac{\partial f_{p}}{\partial y}\right)^{2}
\end{array}\right]
$$

where $n$ represents the number of color planes and $f(x, y)=G_{k} * I(x, y)$ (for a standard color image $\mathrm{n}=3$, i.e. $\left.f=\left[f_{1}, f_{2}, f_{3}\right]\right)$, and $\left(\frac{\partial}{\partial x}, \frac{\partial}{\partial y}\right)$ are the partial derivatives in the horizontal and vertical directions, respectively. The next step involves the eigenvector decomposition of the matrix $M_{k}(x, y)$ to determine the eigenvalues $\lambda_{1}{ }^{k}$ and $\lambda_{2}{ }^{k}, \lambda_{1}{ }^{k} \geq \lambda_{2}{ }^{k}$ (since matrix $M_{k}(x, y)$ is symmetric both eigenvalues are real). If $\lambda_{1}{ }^{k}>>\lambda_{2}{ }^{k}$ and $\lambda_{1}{ }^{k}>>0$, then the pixel is an edge and the multi-scale edge response is calculated as follows,

$$
E(x, y)=\int_{k \in[0, s]} \operatorname{sqrt}\left(\lambda_{1}^{k}\right) d k, \quad \lambda_{1}^{k}=\max \left\{\operatorname{eig}\left[M_{k}(x, y)\right]\right\}
$$

As indicated in equation (5), the edge response calculated for each pixel in the image has only positive values that represent the magnitude of the derivative along the principal direction, and this generates a problem when the edge response $E$ replaces $\nabla I$ in the calculation of the derivative of the flux function (see equation 1). This is motivated by the fact that the values of the pixels resulting after each iteration would follow a monotonic ascent irrespective of the values of the neighboring pixels. 
Proposition: If the gradient $\nabla I$ in equation (1) is replaced with the edge response calculated with equation (5), the intensity value of the pixel $(x, y)$ in image $I$ follows a monotonic ascent with the increase in the number of iterations.

Proof: The Perona-Malik equation shown in (1) can be implemented in the two-dimensional (2D) discrete domain as follows,

$$
I(x, y, t+1)=I(x, y, t)+\frac{\Delta t}{\Delta x \Delta y} \sum_{m \in \Phi(x, y)} D\left(\left|\nabla_{m} I(x, y)\right|\right) \nabla_{m} I(x, y)
$$

where $\Delta t \in \mathfrak{R}^{+}$is the time step, $\Delta x>0, \Delta y>0$ are the discrete spatial distances of the two-dimensional image grid, $t$ is the iteration index, $\nabla_{m} I(x, y)=I(x, y)-I(m) \mid m \in \Phi(x, y), D(.) \in(0,1]$ is the diffusion function and $\Phi(x, y)$ defines the four connected neighborhood of the pixel $(x, y)$. If we replace the gradient $\nabla_{m} I(x, y)$ with $E_{m}(x, y)$ in equation (6) and $E_{m}(x, y) \geq 0$, then,

$$
I(x, y, t+1) \geq I(x, y, t)
$$

To address the inconvenience that is associated with the calculation of the multi-scale edge response $E_{m}(x, y)$, in our approach the value of the edge response $E_{m}$ is multiplied by the sign of the gradient to locally preserve the direction of variation in the image intensity data. This is implemented using the following expression,

$$
E_{m}(x, y) \leftarrow\left[E_{m}(x, y) \times \operatorname{sgn}\left(\nabla I_{m}(x, y)\right)\right], m \in \Phi(x, y)
$$



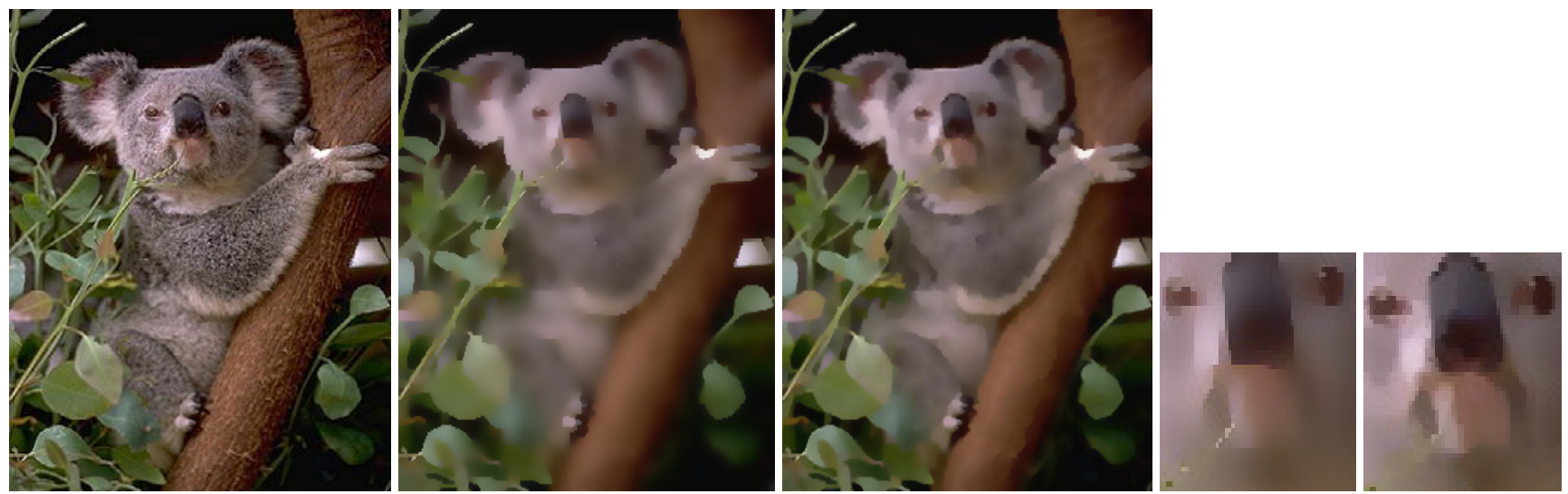

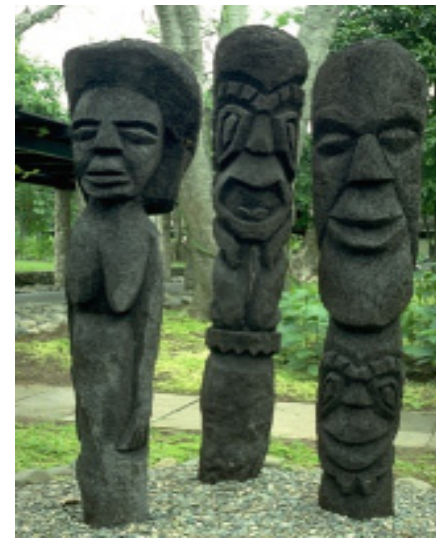

(a)

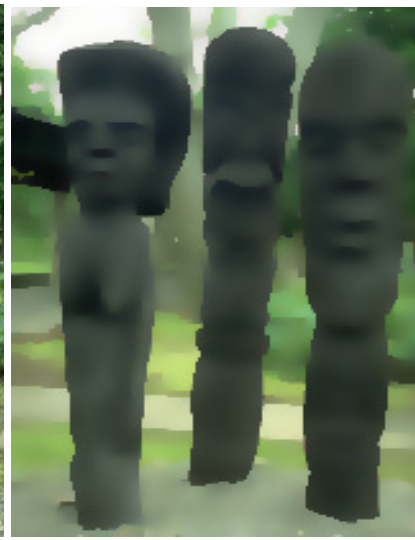

(b)

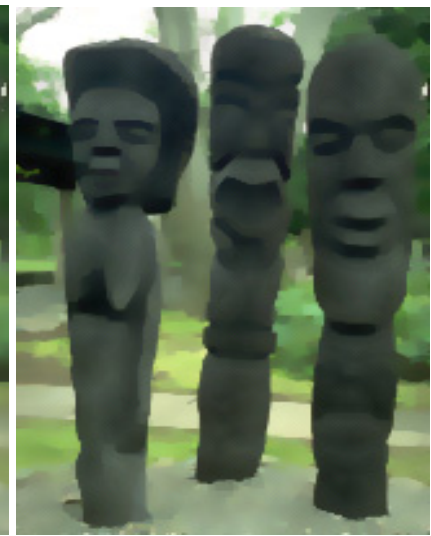

(c)

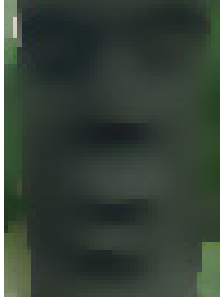

(d)

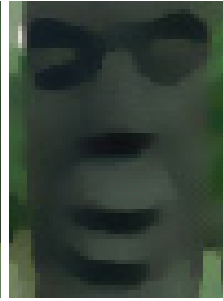

(e)

Fig. 1. The results returned by the standard (PM) algorithm and the proposed multi-scale anisotropic diffusion (MS-AD) when applied to standard test images. (a) Original images [20]. (b) Standard PM anisotropic diffusion results [3]. (c) Multi-scale anisotropic diffusion results. (d) Close-up details from (b). (e) Close-up details from (c).

Fig. 1 depicts the results obtained when the original anisotropic diffusion (AD), as implemented by Perona and Malik [3], and the proposed multi-scale AD are applied to standard test images [20]. The experimental results indicate that the proposed multi-scale AD formulation outperforms the original PM$\mathrm{AD}$ and the advantage associated with our implementation is especially noticeable in areas adjacent to medium gradients. In all experiments the diffusion parameter $d$ has been set to 10.0 and the algorithms were iterated 50 times. The multi-scale edge detector and the multi-scale diffusion function have been calculated for scales in the interval $[0,3.0]$ where the difference between two consecutive scales is 0.5 . In our experiments we have restricted the range of scales in the interval $[0,3.0]$ since the gradient information extracted from images that are convolved with a Gaussian function whose scale is set above 
3.0 has a negligible effect in the calculation of the multi-scale edge detector that is detailed in Section 2.1. This is motivated by the fact that at high-blurring scales the gradient information present in the image is substantially attenuated and it has no tangible effects in the preservation of the image features that are generated by medium gradients.

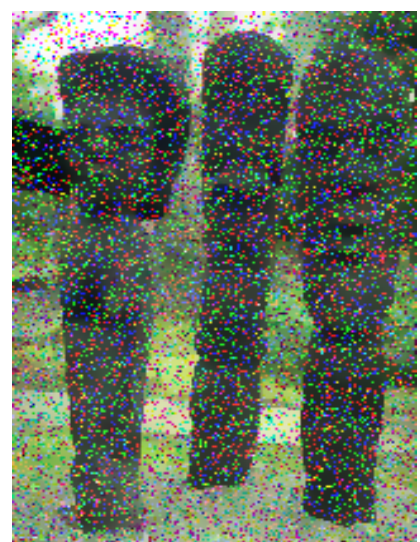

(a)

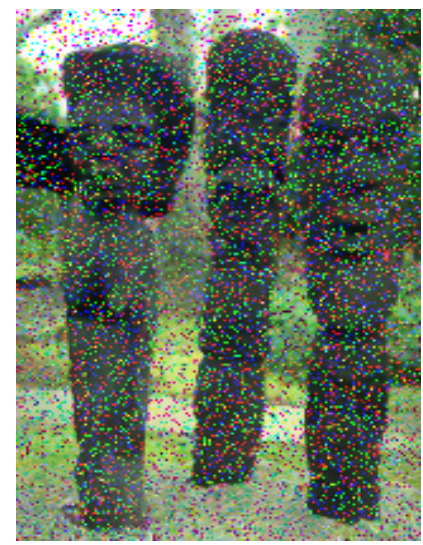

(d)

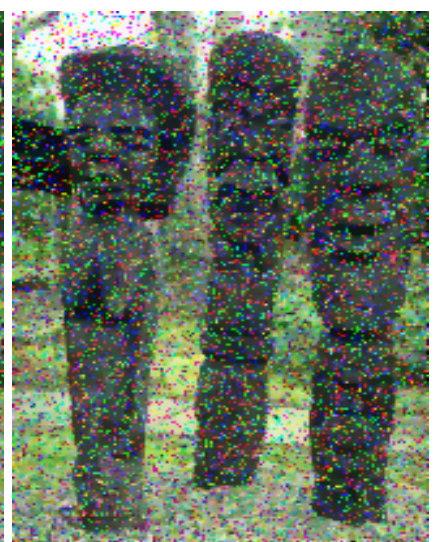

(b)

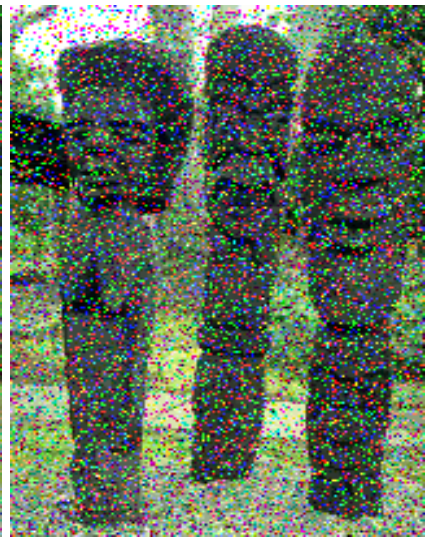

(e)

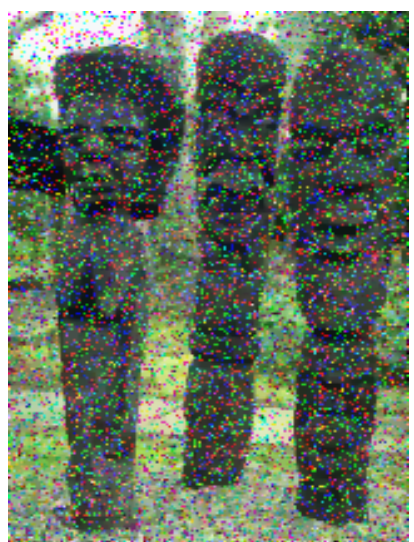

(c)

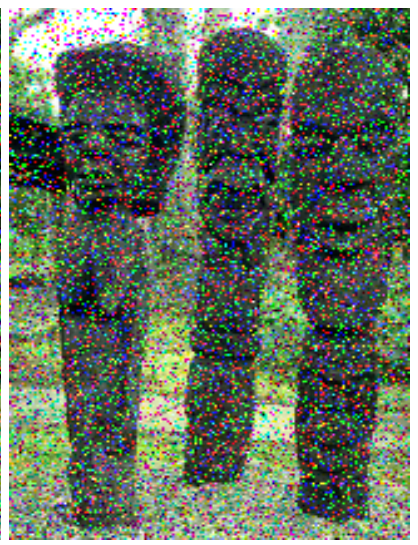

(f)

Fig. 2. The results returned by well-known feature-preserving noise removal strategies when applied to the image shown in (f). (a) PM anisotropic diffusion $(d=10)$ [3]. (b) Total variation flow $(\Delta \mathrm{t}=0.1, \beta=0.1)$ [21]. (c) Bilateral filtering $\left(\sigma_{\mathrm{d}}=0.5, \sigma_{\mathrm{r}}=30\right)$ [22]. (d) Non-Local Means ( $\left.t=7, f=1, h=30\right)$ [23]. (e) Mean shift filtering $\left(\sigma_{\mathrm{s}}=8, \sigma_{\mathrm{r}}=4\right)$ [24]. (f) Original image corrupted by impulse noise (probability $=0.2$ ).

While the inclusion of the gradient information in the formulation of the anisotropic diffusion process is desirable as it allows the implementation of feature-preserving noise removal schemes, on the other hand is detrimental, as it introduces instabilities around pixels that are generated by impulse noise. This 
problem forms one of the major drawbacks associated with all feature-preserving noise removal algorithms that incorporate either partial differential equation (PDE) models or pixel similarity constraints in the data smoothing process and is not restricted only to anisotropic diffusion schemes. This is demonstrated in Fig. 2 where experimental results returned by a number of feature preserving noise removal techniques (when applied to an image corrupted by impulse noise) are illustrated.

\section{Proposed Method}

Based on the observation that the noise removal techniques that incorporate PDE models in the data smoothing process produce inaccurate results when applied to data corrupted by impulse noise, in this paper we propose the implementation of a multi-phase smoothing algorithm where the key issue is the identification of the pixels that are generated by non-Gaussian distributed noise.

In this regard, we attempt to combine two different noise removal strategies in order to implement a data smoothing scheme that exploits the advantages associated with each smoothing strategy. To this end, the anisotropic diffusion filtering and the Vector Median Filtering (VMF) are adaptively combined in order to produce a robust filtering algorithm that is able to return improved performance when applied to data corrupted by multimodal noise.

\subsection{Multimodal noise model}

The overall image distortions can be mathematically expressed by the following formulation,

$$
I(x, y)=F(x, y) * h+\eta(x, y),(x, y) \in \Gamma \subset \mathbf{R}^{2}
$$

where $I$ is the observed image, $F$ denotes the noiseless image, $h$ defines the function that implements the optical distortions and out of focus effects, $\eta(x, y)$ is the noise function and * is the convolution operator. Since the errors inserted by optical distortions do not functionally depend on the image acquisition process, they are typically compensated for by resorting to image transforms that implement inverse radial distortions. Thus, in this paper we aim to correct the errors caused by image noise, while the errors 
inserted by the optical equipment are disregarded. The noise model used in this paper assumes that the data is corrupted by Gaussian and impulse noise, as this noise model accurately approximates the errors generated by the quantum noise in CCD and CMOS sensing elements, the errors that are caused by the sensor and lens dust and the errors introduced by the electronic transmission of the image data. It is useful to note that the errors inserted by the sensing device are accurately modelled by the Gaussian distributed noise, while the latter errors caused by dust or data transmission are well approximated by the impulse noise. Based on the fact that the function $\eta(x, y)$ is defined by two noisy components, namely the Gaussian and impulse noise, the image formation process can be expressed as follows,

$$
I(x, y)= \begin{cases}F(x, y)+\mathcal{N}(0, \sigma) & \text { with probability } 1-\alpha \\ \sup _{(x, y) \in \Gamma} F(x, y) & \text { with probability } \alpha / 2 \\ \inf _{(x, y) \in \Gamma} F(x, y) & \text { with probability } \alpha / 2\end{cases}
$$

where $\mathcal{N}(0, \sigma)$ is the Gaussian noise with zero mean and standard deviation $\sigma, \alpha$ is the probability of the impulse noise, sup and inf are the supremum and infimum functions, respectively, and $\Gamma$ is the $\mathbf{R}^{2}$ image domain. As illustrated in equation (10) the strength of the noise component is controlled by two uncorrelated parameters, $\sigma$ and $\alpha$, and to generate a realistic corruption with multimodal noise, in our work each noise component has been independently applied on each color channel of the original image data. In the remainder of this section we will introduce the VMF scheme and the discussion will be continued with the description of the proposed image restoration technique that integrates the multiscale anisotropic diffusion (MS-AD) and the VMF into an adaptive filtering strategy.

\subsection{Vector Median Filtering}

The vector median filtering (VMF) represents a generalisation of the standard median filtering and has been initially introduced by Astola et al [12]. VMF attempts to minimise the distances between the intensities of the pixels situated within a predefined neighbourhood in order to implement the impulse 
noise suppression. Let $I(x, y)$ be the intensity of the pixel at location $(x, y)$ in the image $I$. The first step of the VMF filtering scheme evaluates the distances between the intensity values of the pixels situated in a neighborhood $\Psi$ around the central pixel $(x, y)$ as follows,

$$
L_{\forall(p, q) \in \Psi}=\sum_{(m, n) \in \Psi}\|I(p, q)-I(m, n)\|
$$

where \|..| defines the $\mathrm{L}_{2}$ norm. The VMF performs the ordering of the $L_{p q}$ values and the intensity of the central pixel of the neighbourhood $\Psi, I(x, y)$, is replaced by the intensity of the pixel that returns the minimum value in the $\left\{L_{p q} \mid(p, q) \in \Psi\right\}$ set.

$$
\left.I(x, y)=\underset{(p, q) \in \Psi}{I(\arg \min }\left(L_{p q}\right)\right)
$$

\subsection{Integration of the MS-AD and VMF noise removal strategies}

As indicated in Section 1, the AD formulation is able to preserve edges during denoising, but is not able to produce accurate results when dealing with images corrupted by impulse noise. Conversely, the VMF scheme has been specifically designed to eliminate the impulse noise, but the denoising process is achieved at the expense of weak feature preservation. In order to exploit the favourable properties associated with both $\mathrm{AD}$ and VMF filtering schemes, we propose the development of a switch mechanism that is able to adaptively select the noise removal strategy for each pixel in the image. If we examine equation (10) in detail, the error between the observed image and the noiseless image can be defined as follows,

$$
e(x, y)=\|I(x, y)-F(x, y)\|=\left\{\begin{array}{cl}
\mathcal{N}(0, \sigma) & \text { with probability } 1-\alpha \\
\left\|F^{*}-F(x, y)\right\| & \text { with probability } \alpha
\end{array}\right.
$$


where $F^{*}=\left\{\sup _{(x, y) \in \Gamma} F(x, y), \inf _{(x, y) \in \Gamma} F(x, y)\right\}$ denotes the set of extreme values associated with the noiseless image $F$ that is defined on the image domain $\Gamma$. Since the errors $\left\|F^{*}-F(x, y)\right\|$ dominate at locations where the pixels in the observed image are corrupted by impulse noise, then the switch mechanism can be implemented as an operator that responds strongly if the pixels situated in a neighbourhood $\Omega(\Omega \subset \Gamma)$ do not approximate a Gaussian distribution. Based on this observation, in the proposed noise removal scheme the impulse noise estimator $\left(N_{e}\right)$ has been implemented as follows,

$$
N_{e}=\left\|I\left(x_{c}, y_{c}\right)-\frac{1}{\operatorname{size}(\Omega)-1} \sum_{(x, y) \in \Omega \backslash\left(x_{c}, y_{c}\right)} I(x, y)\right\|
$$

where $\left(x_{c}, y_{c}\right)$ defines the coordinate of the centre of the neighborhood $\Omega$ and $\operatorname{size}(\Omega)$ denotes the cardinality of the neighborhood $\Omega$. The noise estimator is calculated for each pixel in the image and if the value of $N_{e}$ is higher than a predefined threshold $\xi\left(N_{e} \geq \xi\right)$ then the pixel is assumed to be corrupted by non-Gaussian noise. In our algorithm the output of the noise estimator implements the decision rule that selects the noise removal technique that is applied to each pixel in the image. In this regard, if the value $N_{e}$ calculated for the pixel $(x, y)$ is higher than the predefined threshold $\xi$, then the value of the pixel under observation will be updated using the VMF filtering scheme. Otherwise it will be updated using the proposed multi-scale $\mathrm{AD}$ approach that has been detailed in Section 2. Experimentally, we have determined that the value of the threshold parameter $\xi$ should be set in the interval $[140,260]$. Within this range of values the proposed noise removal strategy returns optimal performance. As illustrated in Fig. 3, for values above the specified range, the efficiency of the proposed algorithm in rejecting the impulse noise decreases. If the value of the threshold parameter is set to values lower than 140 , then the intensity value of the majority pixels in the image will be updated using the VMF component of the proposed algorithm and the smoothing will be achieved at the expense of weak feature preservation. In our implementation the parameter $\xi$ has been set to 160 and this value has been used in all experiments. Note 
that the threshold parameter $\xi$ is not dependent on the probability of the impulse noise, as the noise estimator $N_{e}$ samples the intensity difference between the central pixel of the neighborhood $\Omega$ and the averaged value of its neighboring pixels (see equation 14).

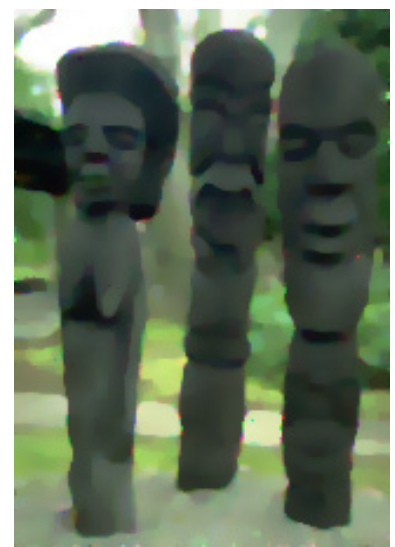

(a)

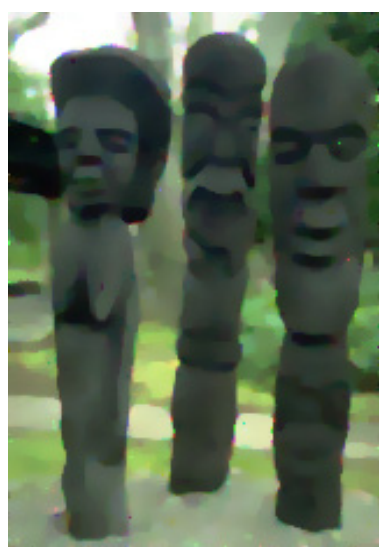

(b)

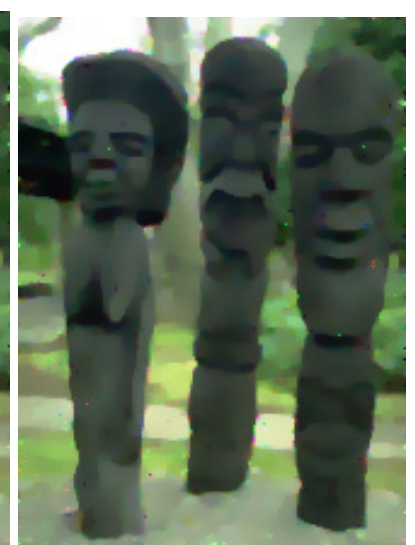

(c)

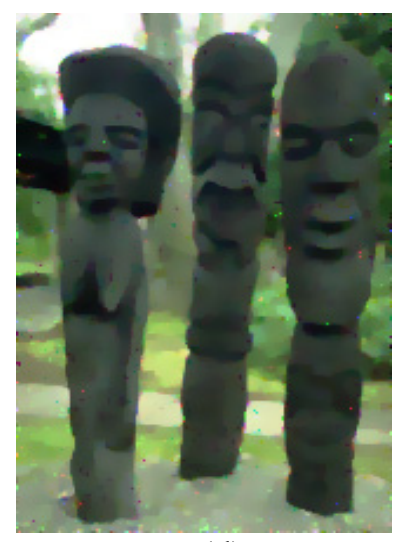

(d)

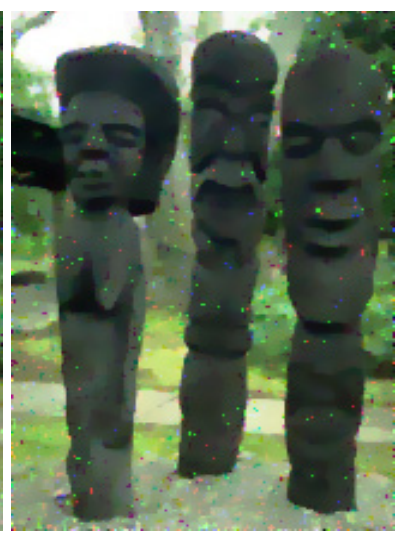

(e)

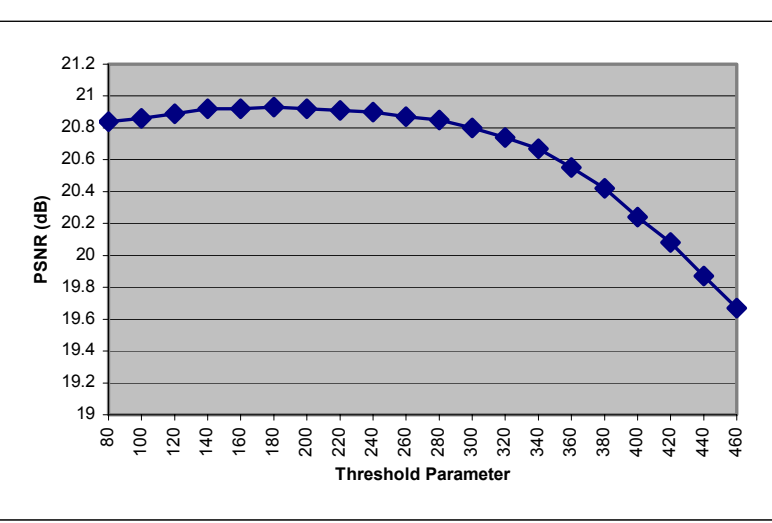

(f)

Fig. 3. The performance of the algorithm with respect to the variation of the $\xi$ parameter when applied to the image depicted in Fig. 2(f) (PSNR $=11.43 \mathrm{~dB})$. (a) $\xi=120$. (b) $\xi=160$. (c) $\xi=200$. (d) $\xi=260$; (e) $\xi=400$; (f) PSNR values when the parameter $\xi$ is varied in the interval $[80,460]$.

\section{Experimental Results}

In this study we evaluate the performance of the proposed data smoothing technique when applied to the task of denoising color images. In order to achieve this goal, the original images were corrupted by 
multi-component (multimodal) noise and the performance of the proposed filtering scheme is quantitatively evaluated using the peak-signal-to-noise-ratio (PSNR).

$$
\operatorname{PSNR}=10 \log _{10} \frac{\max _{(x, y) \in \Gamma}(I(x, y))^{2}}{\frac{1}{\operatorname{size}(\Gamma)} \iint\|O(x, y)-I(x, y)\|^{2} d x d y}
$$

where $\Gamma \subset \mathbf{R}^{2}$ is the image domain, $O(x, y)$ defines the pixel intensities of the original image $O$ and $I(x, y)$ are the pixel intensities resulting after the data smoothing algorithms were applied to the image that was corrupted by multimodal noise. Since the PSNR does not accurately sample the efficiency of the noise suppression process with respect to edge preservation, to complement this measure, an additional edge preservation index (Epi) that was suggested in [27] was also quantified.

$$
E p i=\frac{\Lambda(O-\bar{O}, I-\bar{I})}{\sqrt{\Lambda(O-\bar{O}, O-\bar{O}) \Lambda(I-\bar{I}, I-\bar{I})}}
$$

where $O$ is the original image, $I$ defines the image resulting from the smoothing process, $\bar{O}$ and $\bar{I}$ are the mean intensity values calculated from images $O$ and $I$, respectively, and $\Lambda(q, r)=\sum_{(x, y) \in \Gamma} q(x, y) r(x, y)$. The Epi index is normalised in the interval $[0,1]$ and a value closer to 1 indicate an accurate edge preservation attained by the data smoothing algorithm. Additionally, to quantify the perceptual color difference between the original image $O$ and the output returned by the data smoothing algorithms $I$, we have calculated the CIEDE2000 $\left(\Delta \mathrm{E}_{00}\right)$ index that has been detailed in [31,32]. The $\Delta \mathrm{E}_{00}$ index has been normalised with respect to the image size and a value $\Delta \mathrm{E}_{00}=0$ indicates no color difference between images $O$ and $I$. In our experimental tests we have generated results when the performance of the proposed algorithm has been compared against those offered by the MS-AD, VMF3 and VMF5 techniques. To provide further performance evaluation details, the accuracy attained by the data smoothing strategy introduced in this paper has been also benchmarked against those achieved by two recently proposed state 
of the art image restoration methods that have been specifically designed to address the noise removal in images corrupted by Gaussian and impulse noise. Thus, the method proposed by Yang and Fox [29] (denoted as PM-Median) performs a weighted sum between the output generated by the standard PM anisotropic function and the median filter, while in the approach proposed by Ghita and Whelan [28],

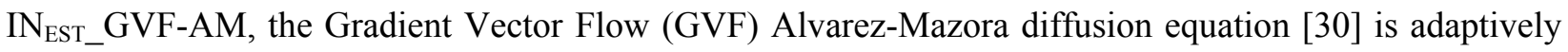
coupled with the response of the median filter. It is useful to mention that in the implementation of these image restoration strategies the anisotropic models are coupled with the median filter and the output will always be a combination of these two noise reduction components. Contrary to this approach, in the image restoration strategy proposed in this paper a switch mechanism that responds strongly to data corrupted by non-Gaussian noise has been implemented to select the appropriate noise reduction technique, a solution that we envision has clear advantages in terms of improved feature preservation. One additional disadvantage associated with approaches based on coupling the anisotropic models and median filters is worth noticing - the intensity shifts that are introduced by the coupling process. In the experimental section, for consistency reasons, we will report the PSNR results for the methods proposed in [28, 29] for two scenarios, with (PSNR_ISC) and without (PSNR) intensity shifts (offsets) correction.

The first set of experiments was conducted on test images corrupted by multimodal noise. In this sense, we have generated images that were corrupted by both additive Gaussian and impulse noise and experimental results are shown in Figs. 4 to 7 . The experimental results depicted in Figs. 4 to 7 indicate that the MS-AD and VMF3 algorithms are clearly outperformed by the proposed and the VMF5 algorithm and in all experiments the proposed image restoration scheme outperformed the VMF5 algorithm when the examined noise removal schemes are evaluated using the PSNR metric. For the sake of completeness, additional numerical results including the edge preservation index (Epi) (see Eq. 16) and $\Delta \mathrm{E}_{00}$ index are included in Tables 1 and 2 to complement the PSNR values. 


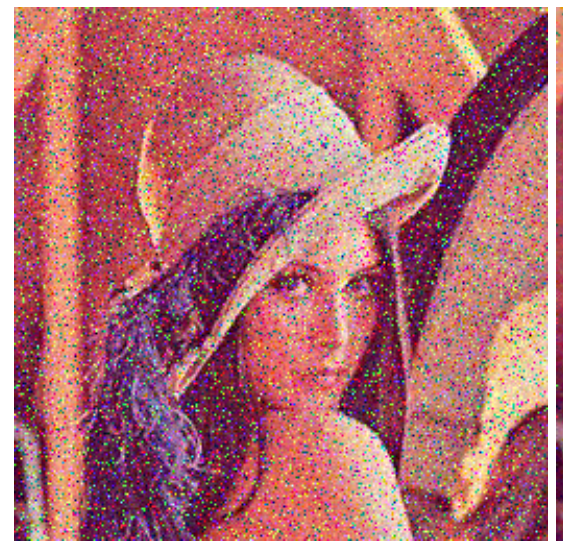

(a)

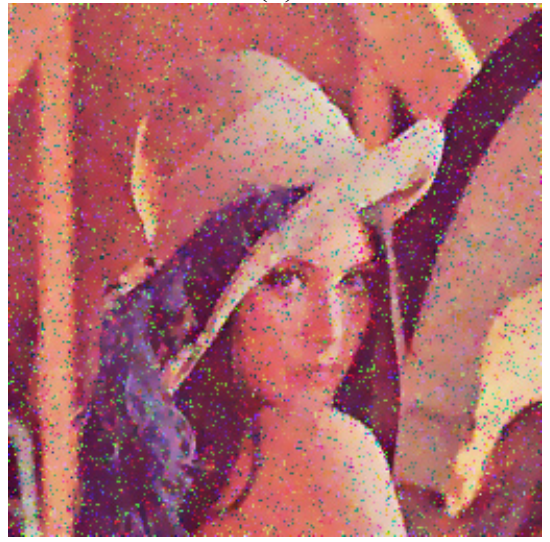

(d)

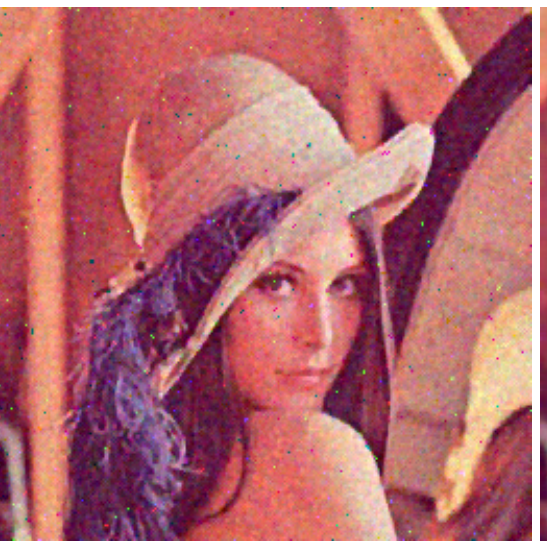

(b)

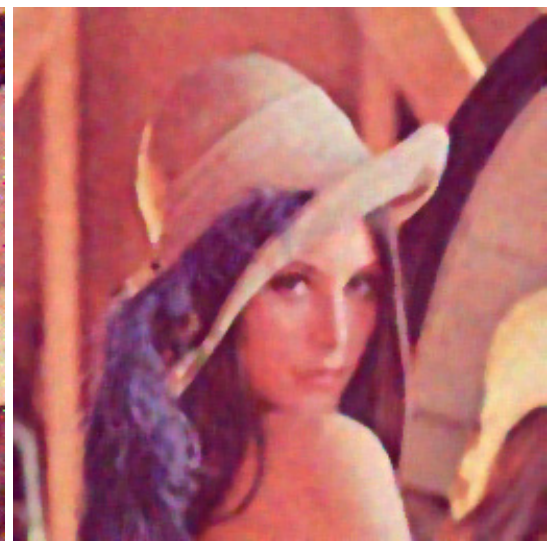

(c)

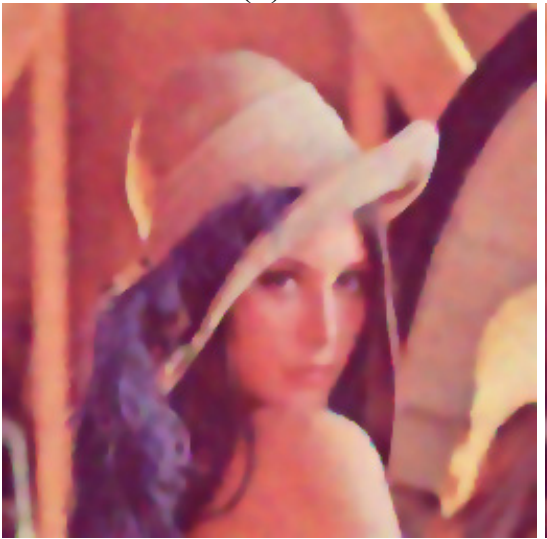

(e)

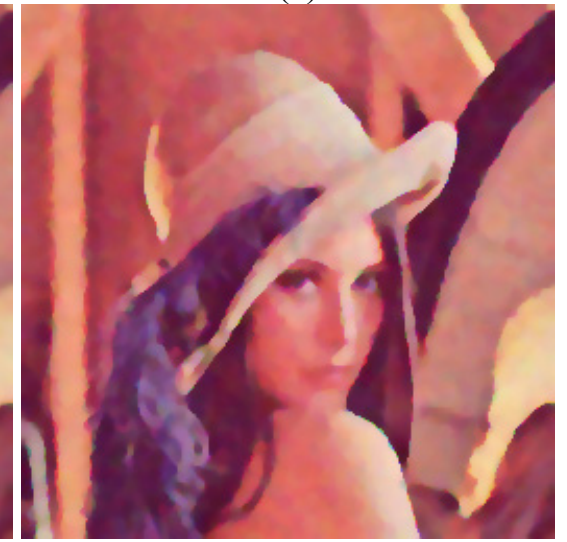

(f)

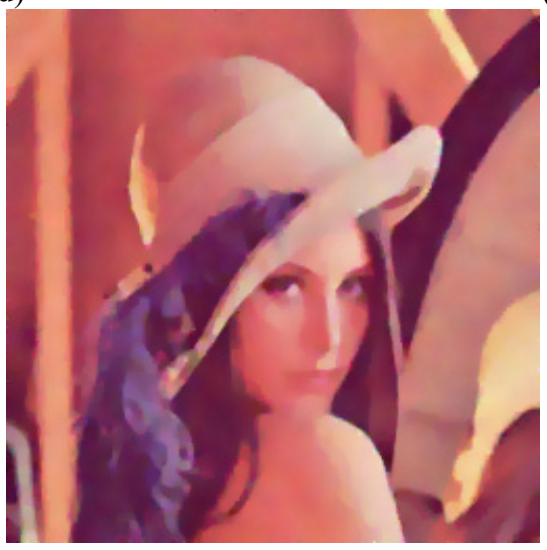

(g)

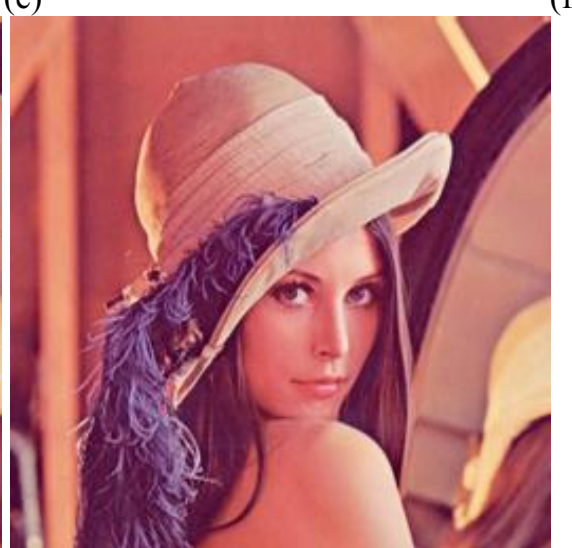

(h)

Fig. 4. Experimental results in the presence of multimodal noise - Lena image. (a) Test image corrupted by Gaussian noise $(\mathcal{N}(0,20)$ ) and impulse noise - probability 0.1. (b) Vector Median Filter (window $\Psi=$ $3 \times 3-$ VMF3). (c) Vector Median Filter (window $\Psi=5 \times 5-$ VMF5). (d) Multi-scale anisotropic diffusion (MS-AD). (e) PM-Median. (f) IN $\mathrm{INST}_{-}$GVF-AM. (g) Proposed algorithm (MS-AD-VMF, VMF window size $\Psi=5 \times 5$ ). (h) Original image. 


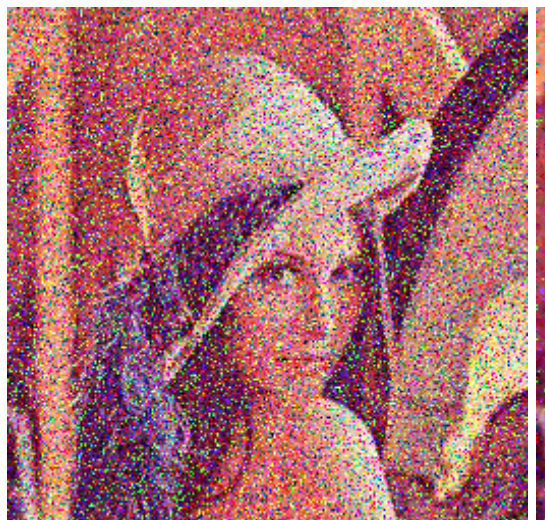

(a)

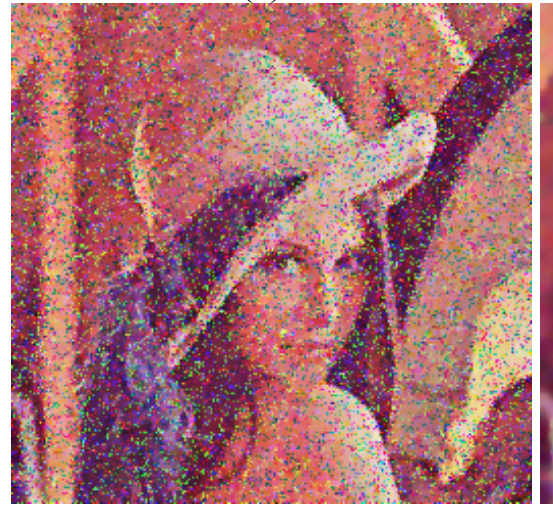

(d)

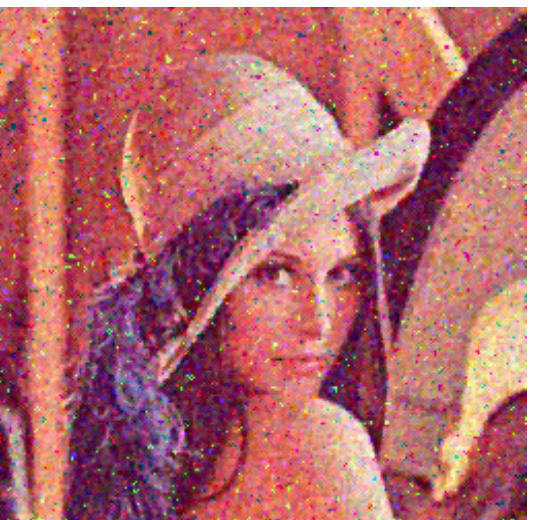

(b)

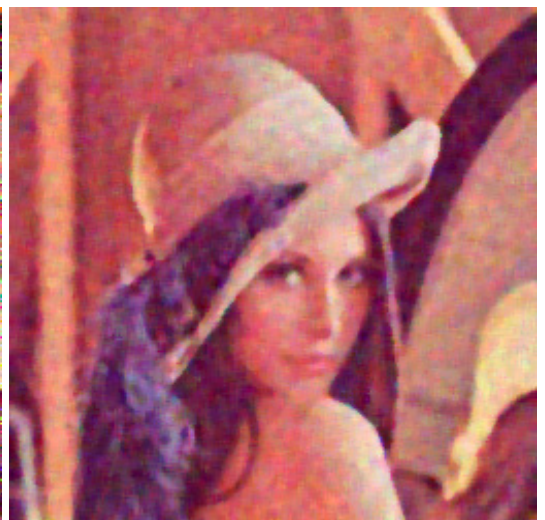

(c)

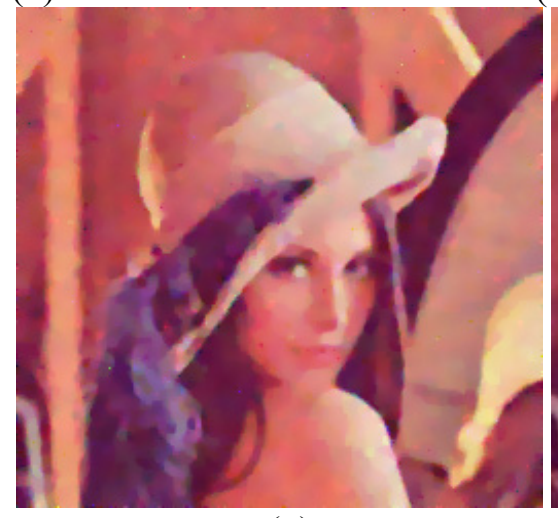

(g)

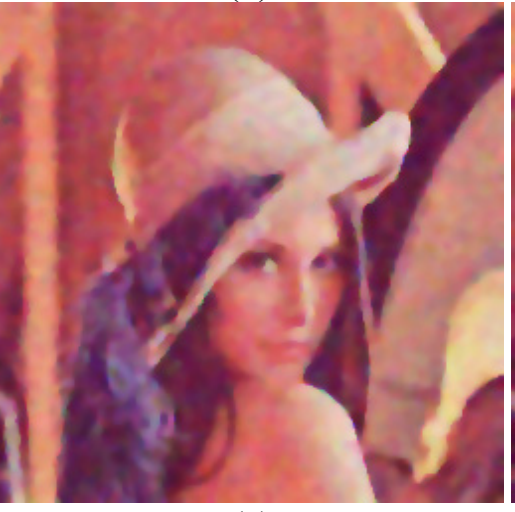

(e)

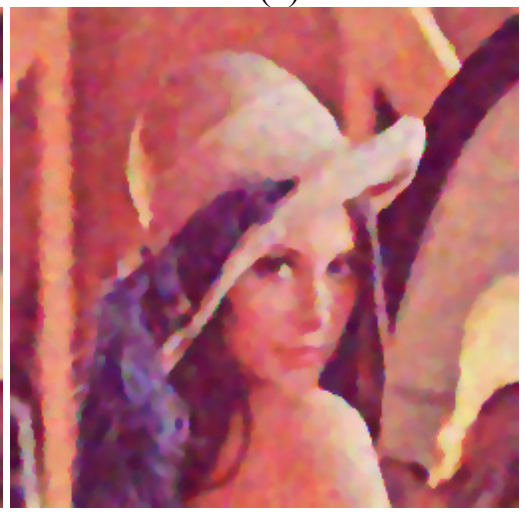

(f)

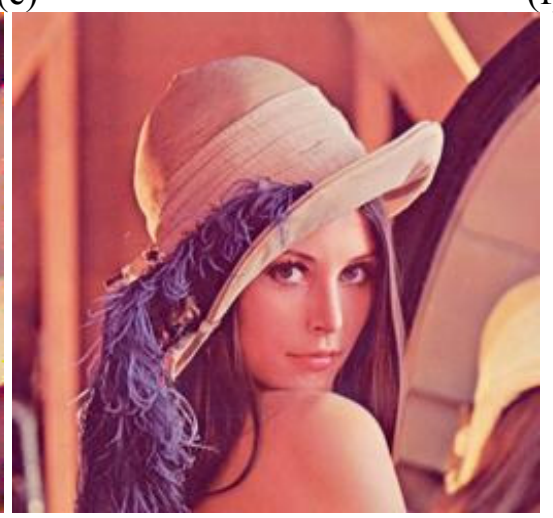

(h)

Fig. 5. Experimental results in the presence of multimodal noise - Lena image. (a) Test image corrupted by Gaussian noise $(\mathcal{N}(0,30)$ ) and impulse noise - probability 0.2. (b) Vector Median Filter (window $\Psi=$ $3 \times 3-$ VMF3). (c) Vector Median Filter (window $\Psi=5 \times 5-$ VMF5). (d) Multi-scale anisotropic diffusion (MS-AD). (e) PM-Median. (f) $\mathrm{IN}_{\mathrm{EST}}$ GVF-AM. (g) Proposed algorithm (MS-AD-VMF, VMF window $\Psi$ $=5 \times 5)$. (h) Original image. 


\begin{tabular}{|c|c|c|c|c|c|}
\hline Method & Noise level & PSNR & PSNR_ISC & Epi & $\Delta \mathrm{E}_{00}$ \\
\hline $\begin{array}{l}\text { VMF3 } \\
\text { VMF5 } \\
\text { MS-AD } \\
\text { IN }_{\text {EST_GVF-AM }} \\
\text { PM-Median } \\
\text { Proposed (MS-AD-VMF) }\end{array}$ & $\mathcal{N}(0,10), \alpha=0.1$ & $\begin{array}{l}26.68 \\
27.28 \\
19.66 \\
20.83 \\
20.58 \\
27.24\end{array}$ & $\begin{array}{c}- \\
- \\
- \\
26.36 \\
26.32 \\
-\end{array}$ & $\begin{array}{l}0.965 \\
0.966 \\
0.832 \\
0.960 \\
0.959 \\
0.966\end{array}$ & $\begin{array}{l}3.50 \\
2.59 \\
6.72 \\
3.00 \\
2.81 \\
2.66\end{array}$ \\
\hline $\begin{array}{l}\text { VMF3 } \\
\text { VMF5 } \\
\text { MS-AD } \\
\text { IN }_{\text {EST_GVF-AM }} \\
\text { PM-Median } \\
\text { Proposed (MS-AD-VMF) }\end{array}$ & $\mathcal{N}(0,20), \alpha=0.2$ & $\begin{array}{l}20.32 \\
25.82 \\
15.99 \\
20.59 \\
20.48 \\
26.11 \\
\end{array}$ & $\begin{array}{c}- \\
25.68 \\
25.49 \\
-\end{array}$ & $\begin{array}{l}0.865 \\
0.953 \\
0.668 \\
0.951 \\
0.951 \\
0.956 \\
\end{array}$ & $\begin{array}{c}7.85 \\
3.90 \\
12.05 \\
3.91 \\
3.71 \\
3.25 \\
\end{array}$ \\
\hline $\begin{array}{l}\text { VMF3 } \\
\text { VMF5 } \\
\text { MS-AD } \\
\text { IN }_{\text {EST_GVF-AM }} \\
\text { PM-Median } \\
\text { Proposed (MS-AD-VMF) }\end{array}$ & $\mathcal{N}(0,30), \alpha=0.3$ & $\begin{array}{l}15.90 \\
23.77 \\
13.13 \\
20.02 \\
19.91 \\
24.50 \\
\end{array}$ & $\begin{array}{c}- \\
- \\
- \\
24.26 \\
23.86 \\
-\end{array}$ & $\begin{array}{l}0.705 \\
0.925 \\
0.491 \\
0.930 \\
0.931 \\
0.936 \\
\end{array}$ & $\begin{array}{c}13.63 \\
5.76 \\
18.48 \\
5.26 \\
5.12 \\
4.47 \\
\end{array}$ \\
\hline $\begin{array}{l}\text { VMF3 } \\
\text { VMF5 } \\
\text { MS-AD } \\
\text { IN }_{\text {EST_GVF-AM }} \\
\text { PM-Median } \\
\text { Proposed (MS-AD-VMF) }\end{array}$ & $\mathcal{N}(0,40), \alpha=0.4$ & $\begin{array}{l}12.91 \\
21.12 \\
11.20 \\
19.26 \\
19.16 \\
22.02\end{array}$ & $\begin{array}{c}- \\
- \\
- \\
22.05 \\
21.60 \\
-\end{array}$ & $\begin{array}{l}0.547 \\
0.870 \\
0.363 \\
0.891 \\
0.889 \\
0.892\end{array}$ & $\begin{array}{c}20.15 \\
8.50 \\
24.26 \\
7.24 \\
7.32 \\
6.83\end{array}$ \\
\hline $\begin{array}{l}\text { VMF3 } \\
\text { VMF5 } \\
\text { MS-AD } \\
\text { IN }_{\text {EST_GVF-AM }} \\
\text { PM-Median } \\
\text { Proposed (MS-AD-VMF) }\end{array}$ & $\mathcal{N}(0,50), \alpha=0.5$ & $\begin{array}{c}10.65 \\
17.97 \\
9.80 \\
18.11 \\
17.88 \\
18.85 \\
\end{array}$ & $\begin{array}{c}- \\
- \\
- \\
20.03 \\
19.35 \\
- \\
\end{array}$ & $\begin{array}{l}0.407 \\
0.773 \\
0.264 \\
0.826 \\
0.815 \\
0.805 \\
\end{array}$ & $\begin{array}{l}26.50 \\
12.59 \\
28.81 \\
10.10 \\
10.51 \\
10.78 \\
\end{array}$ \\
\hline
\end{tabular}

Table 1. Quantitative results reported for all analysed noise removal techniques - Lena image.

The results depicted in Figs. 4 to 7 and Tables 1 and 2 indicate that the proposed image restoration strategy outperforms the VMF and MS-AD noise removal methods due to its ability to locally adapt to the characteristics of the image noise. When the performance of the proposed strategy is contrasted to those obtained by the multimodal noise reduction methods detailed in $[28,29]$ we can observe that our method also returns improved performance except the situation when the image is corrupted with very high levels of impulse noise. Obviously, in this evaluation scenario the advantage of coupling the anisotropic models with the median filter becomes apparent, as the impulse component is dominant in the noise model. 


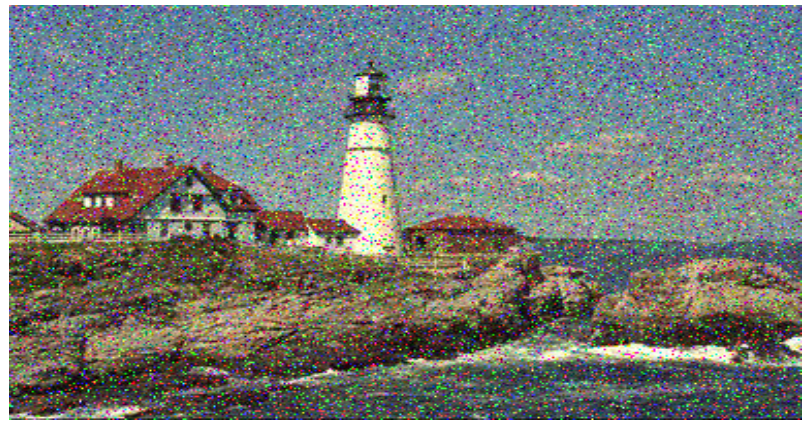

(a)

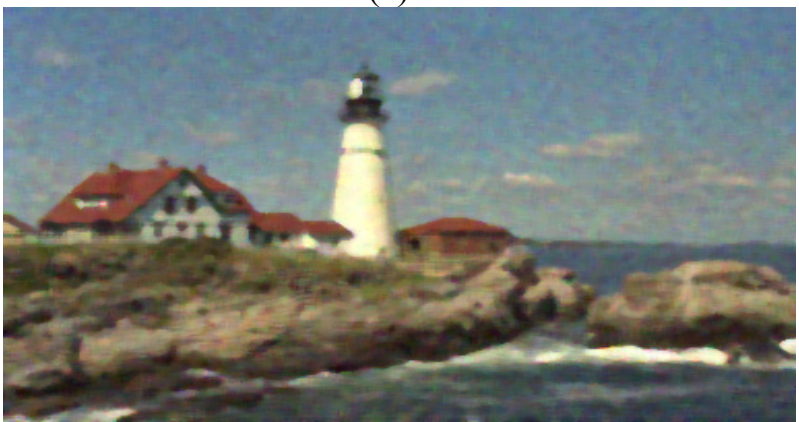

(c)

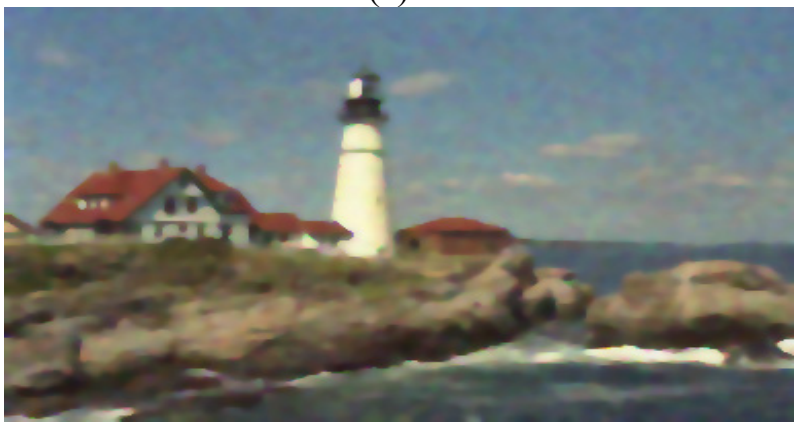

(e)

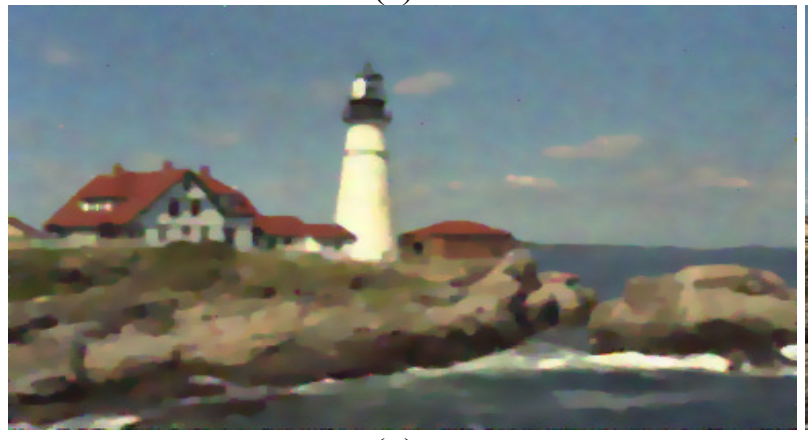

(g)

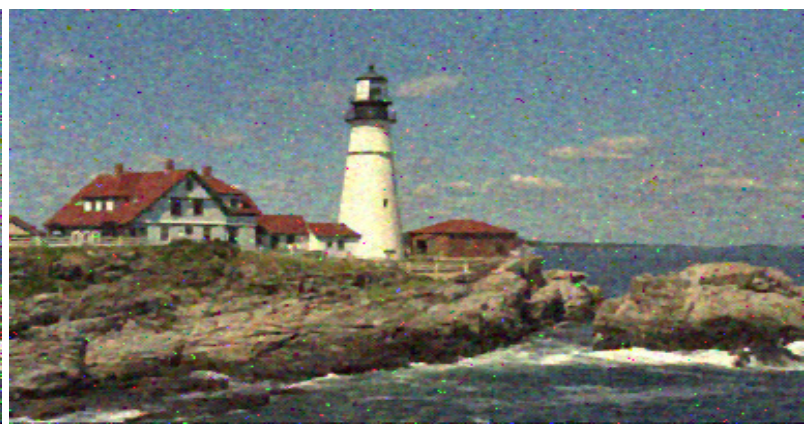

(b)

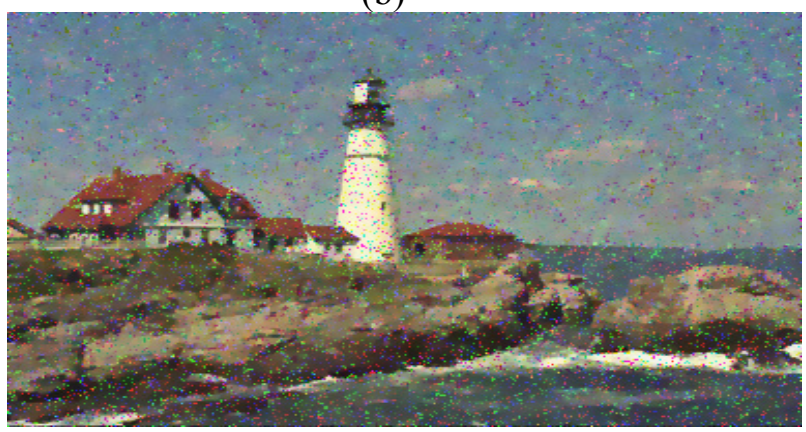

(d)

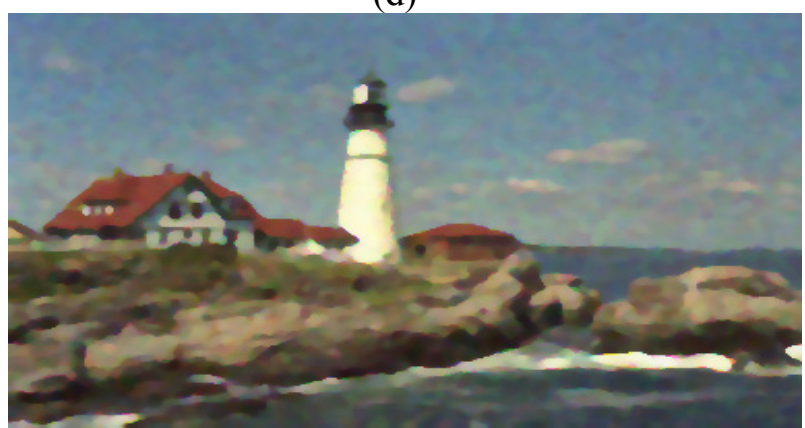

(f)

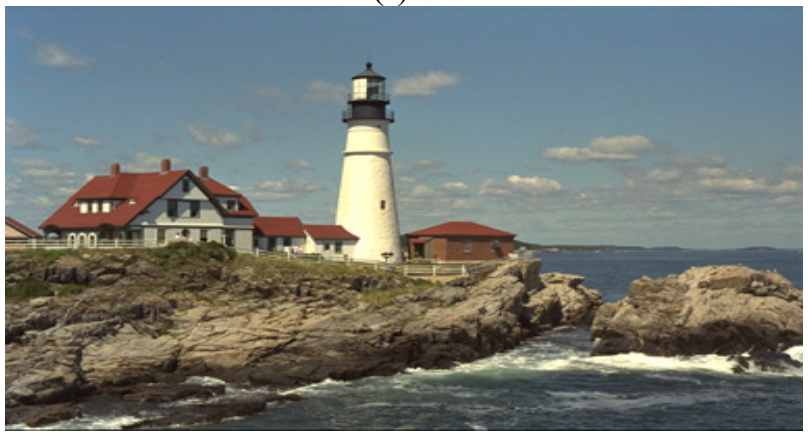

(h)

Fig. 6. Experimental results in the presence of multimodal noise - Kodak image. (a) Test image corrupted by Gaussian noise $(\mathcal{N}(0,20)$ ) and impulse noise - probability 0.1. (b) Vector Median Filter (window $\Psi=$ $3 \times 3$ - VMF3). (c) Vector Median Filter (window $\Psi=5 \times 5-$ VMF5). (d) Multi-scale anisotropic diffusion

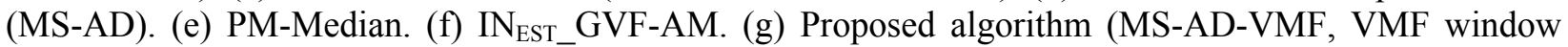
size $\Psi=5 \times 5$ ). (h) Original image. 


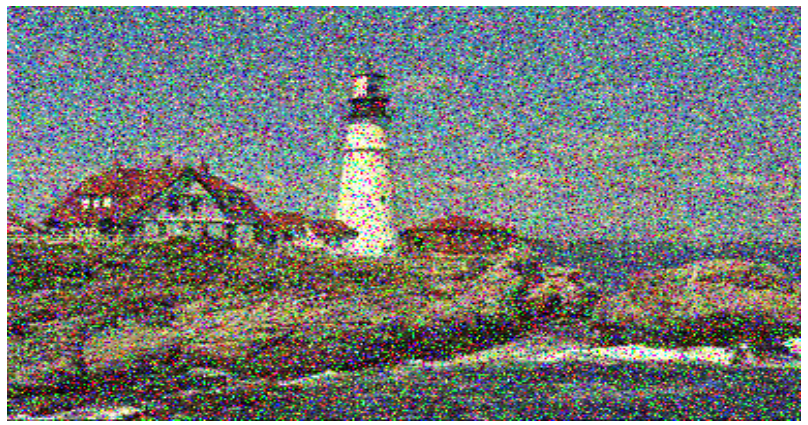

(a)

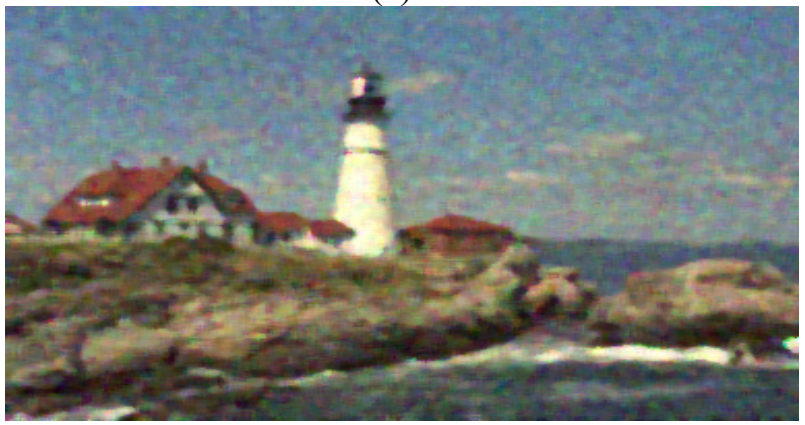

(c)

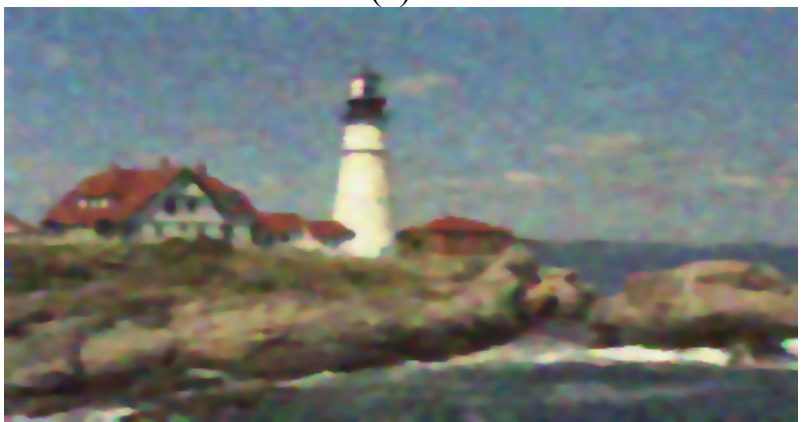

(e)

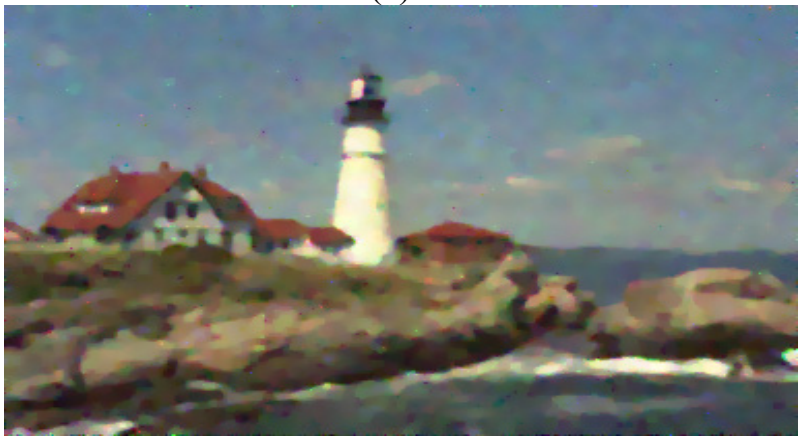

$(\mathrm{g})$

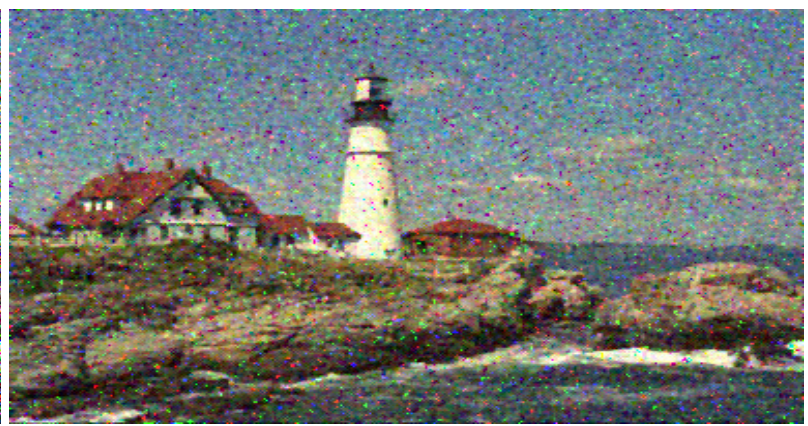

(b)

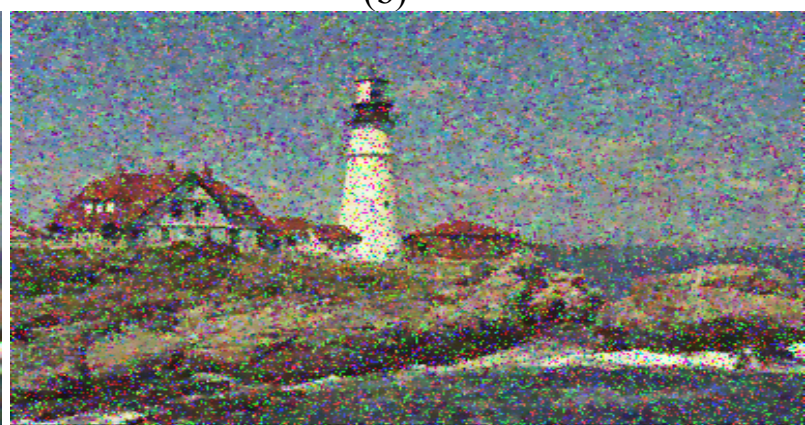

(d)

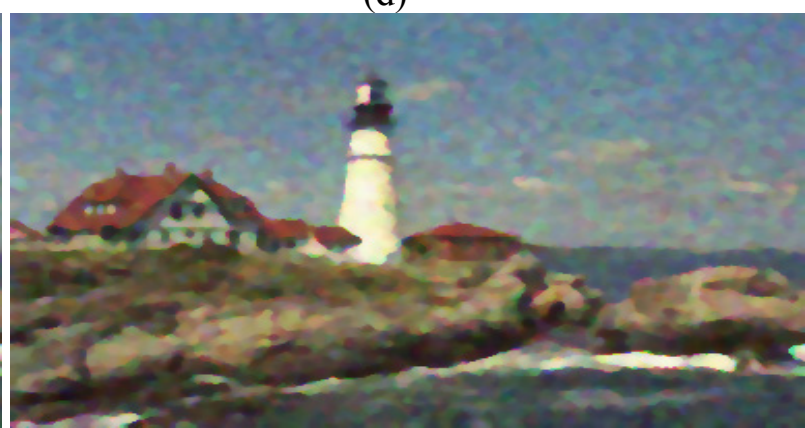

(f)

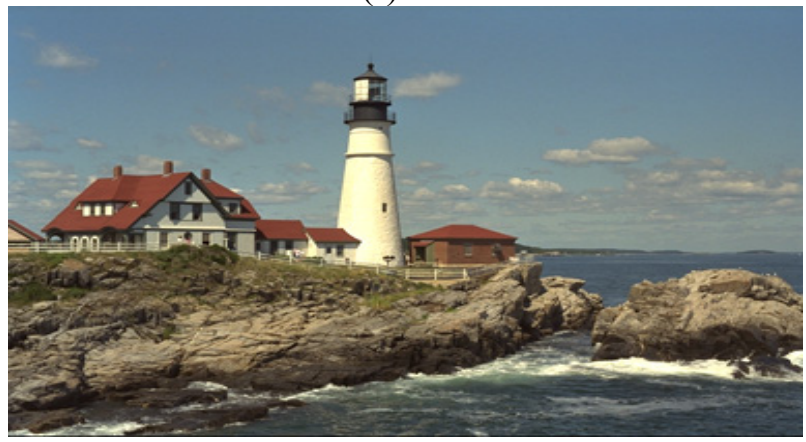

(h)

Fig. 7. Experimental results in the presence of multimodal noise - Kodak image. (a) Test image corrupted by Gaussian noise $(\mathcal{N}(0,30)$ ) and impulse noise - probability 0.2 . (b) Vector Median Filter (window $\Psi=$ $3 \times 3-$ VMF3). (c) Vector Median Filter (window $\Psi=5 \times 5-$ VMF5). (d) Multi-scale anisotropic diffusion (MS-AD). (e) PM-Median. (f) $\mathrm{IN}_{\mathrm{EST}}$ GVF-AM. (g) Proposed algorithm (MS-AD-VMF, VMF window $\Psi$ $=5 \times 5$ ). (h) Original image. 


\begin{tabular}{|c|c|c|c|c|c|}
\hline Method & Noise level & PSNR & PSNR_ISC & Epi & $\Delta \mathrm{E}_{00}$ \\
\hline $\begin{array}{l}\text { VMF3 } \\
\text { VMF5 } \\
\text { MS-AD } \\
\text { IN }_{\text {EST_GVF-AM }} \\
\text { PM-Median } \\
\text { Proposed (MS-AD-VMF) }\end{array}$ & $\mathcal{N}(0,10), \alpha=0.1$ & $\begin{array}{l}26.12 \\
24.61 \\
20.76 \\
23.36 \\
23.07 \\
24.99 \\
\end{array}$ & $\begin{array}{c}- \\
- \\
- \\
24.28 \\
24.16 \\
-\end{array}$ & $\begin{array}{l}0.957 \\
0.938 \\
0.852 \\
0.939 \\
0.938 \\
0.943 \\
\end{array}$ & $\begin{array}{l}5.05 \\
3.66 \\
6.92 \\
3.71 \\
3.47 \\
3.42 \\
\end{array}$ \\
\hline $\begin{array}{l}\text { VMF3 } \\
\text { VMF5 } \\
\text { MS-AD } \\
\text { IN }_{\text {EST_GVF-AM }} \\
\text { PM-Median } \\
\text { Proposed (MS-AD-VMF) }\end{array}$ & $\mathcal{N}(0,20), \alpha=0.2$ & $\begin{array}{l}20.27 \\
23.81 \\
16.81 \\
22.87 \\
22.58 \\
24.24\end{array}$ & $\begin{array}{c}- \\
23.72 \\
23.60 \\
-\end{array}$ & $\begin{array}{l}0.854 \\
0.924 \\
0.678 \\
0.928 \\
0.928 \\
0.932\end{array}$ & $\begin{array}{c}9.96 \\
5.53 \\
12.34 \\
5.17 \\
4.76 \\
4.31\end{array}$ \\
\hline $\begin{array}{l}\text { VMF3 } \\
\text { VMF5 } \\
\text { MS-AD } \\
\text { IN }_{\text {EST_GVF-AM }} \\
\text { PM-Median } \\
\text { Proposed (MS-AD-VMF) } \\
\end{array}$ & $\mathcal{N}(0,30), \alpha=0.3$ & $\begin{array}{l}16.02 \\
22.47 \\
13.72 \\
21.84 \\
21.60 \\
23.16 \\
\end{array}$ & $\begin{array}{c}- \\
- \\
- \\
22.59 \\
22.55 \\
- \\
\end{array}$ & $\begin{array}{l}0.692 \\
0.897 \\
0.494 \\
0.908 \\
0.909 \\
0.913 \\
\end{array}$ & $\begin{array}{c}15.27 \\
7.98 \\
17.88 \\
7.03 \\
6.55 \\
6.05 \\
\end{array}$ \\
\hline $\begin{array}{l}\text { VMF3 } \\
\text { VMF5 } \\
\text { MS-AD } \\
\text { IN }_{\text {EST_GVF-AM }} \\
\text { PM-Median } \\
\text { Proposed (MS-AD-VMF) }\end{array}$ & $\mathcal{N}(0,40), \alpha=0.4$ & $\begin{array}{l}13.02 \\
20.33 \\
11.76 \\
20.49 \\
20.42 \\
21.21 \\
\end{array}$ & $\begin{array}{c}- \\
- \\
- \\
21.08 \\
21.18 \\
-\end{array}$ & $\begin{array}{l}0.532 \\
0.841 \\
0.363 \\
0.865 \\
0.866 \\
0.868 \\
\end{array}$ & $\begin{array}{c}20.33 \\
10.99 \\
22.02 \\
9.39 \\
8.94 \\
8.99 \\
\end{array}$ \\
\hline $\begin{array}{l}\text { VMF3 } \\
\text { VMF5 } \\
\text { MS-AD } \\
\text { IN }_{\text {EST_GVF-AM }} \\
\text { PM-Median } \\
\text { Proposed (MS-AD-VMF) } \\
\end{array}$ & $\mathcal{N}(0,50), \alpha=0.5$ & $\begin{array}{l}10.75 \\
17.36 \\
10.47 \\
18.67 \\
18.54 \\
18.28 \\
\end{array}$ & $\begin{array}{c}- \\
- \\
- \\
19.16 \\
19.16 \\
- \\
\end{array}$ & $\begin{array}{l}0.385 \\
0.728 \\
0.262 \\
0.786 \\
0.778 \\
0.768 \\
\end{array}$ & $\begin{array}{l}24.94 \\
14.77 \\
24.67 \\
12.17 \\
11.86 \\
12.95 \\
\end{array}$ \\
\hline
\end{tabular}

Table 2. Quantitative results reported for all analysed noise removal techniques - Kodak image.

Fig. 8 depicts additional results when the analysed noise removal techniques are applied to strongly textured images. To limit the size of the diagram only the results obtained for VMF3, VMF5, IN $\mathrm{EST}_{\text {_GVF- }}$ AM and proposed image enhancement strategy are presented in Fig. 8, as the performance of the PMMedian is generally lower than that offered by the $\mathrm{IN}_{\mathrm{EST}}$ GVF-AM. These experimental results show that the proposed strategy returns improved performance when visually assessed and when numerically evaluated with respect to PSNR values. 


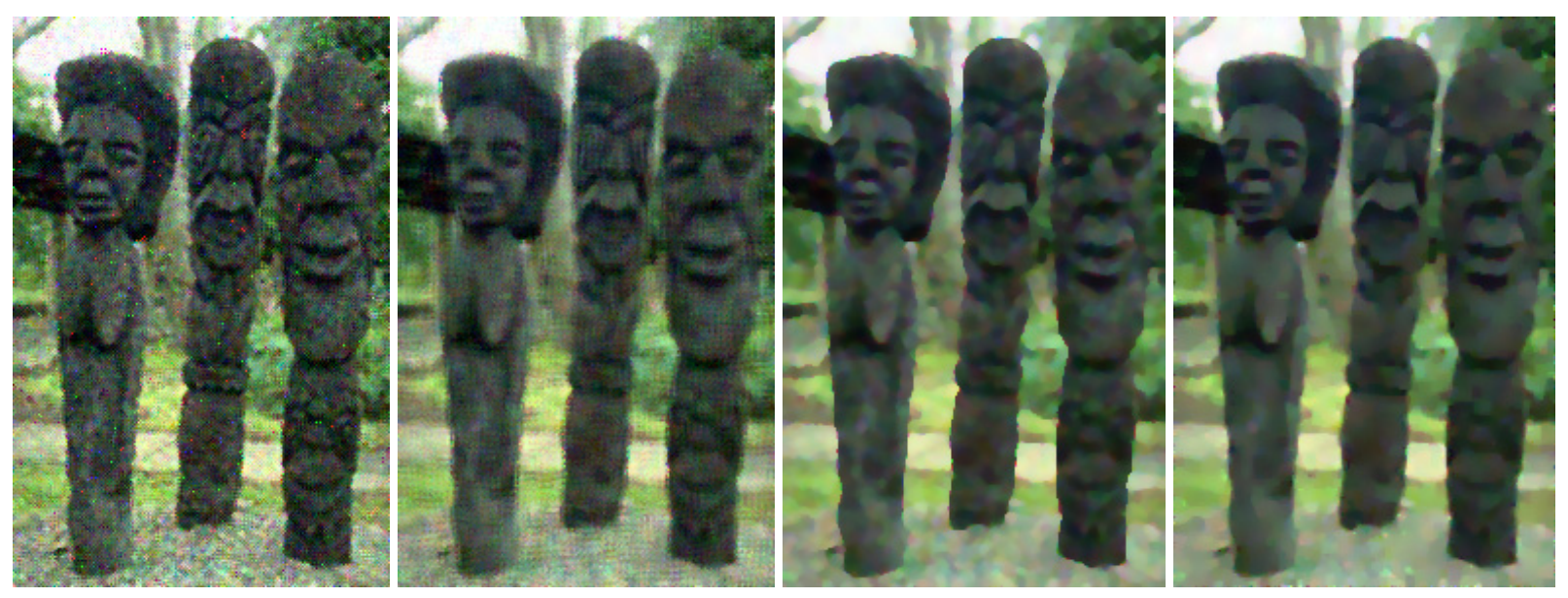

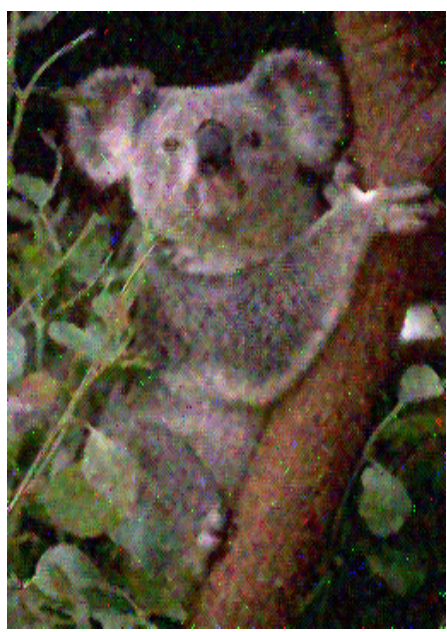

(a)

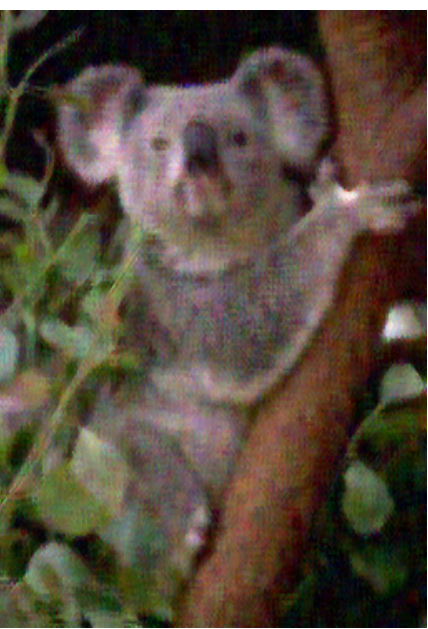

(b)

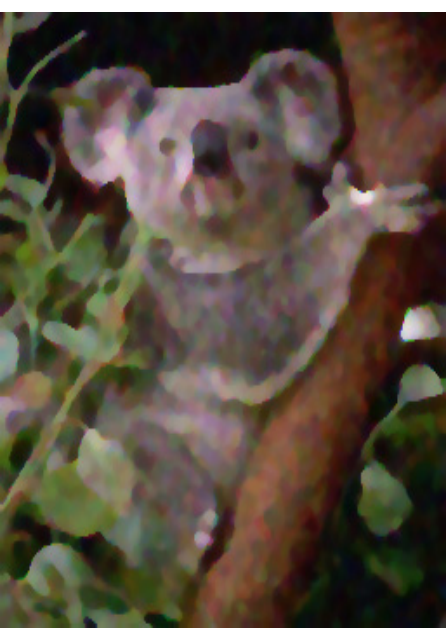

(c)

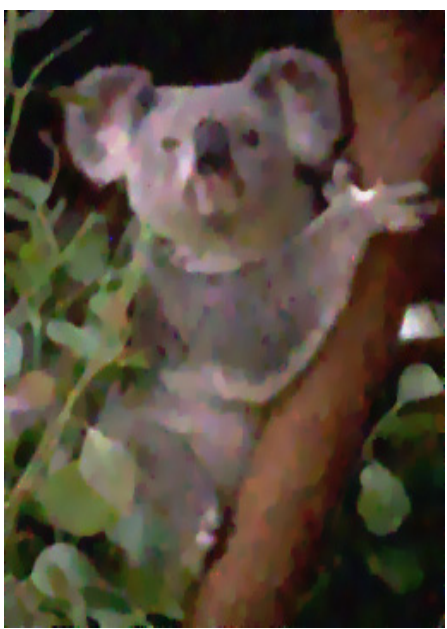

(d)

Fig. 8. Additional experimental results when the examined noise removal strategies were applied to images shown in Fig. 1 that are corrupted by Gaussian $(\mathcal{N}(0,30))$ and impulse noise - probability 0.1. (a) Vector Median Filter (window $\Psi=3 \times 3-$ VMF3). PSNR values: 18.60 - top image, 20.51 - bottom image. (b) Vector Median Filter (window $\Psi=5 \times 5$ - VMF5). PSNR values: 20.58 - top image, $22.04-$

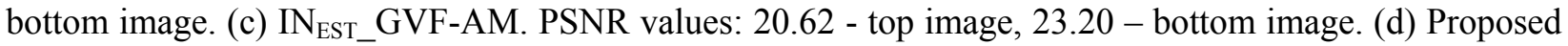
algorithm (MS-AD-VMF, VMF window $\Psi=5 \times 5$ ). PSNR values: 20.75 - top image, 23.31 - bottom image.

The last set of experiments was conducted to evaluate the performance of the noise removal strategies investigated in this paper when applied to the segmentation of medical images that are characterised by low contrast and low signal to noise ratio (SNR). In this regard, we have applied an unsupervised KMeans clustering algorithm (initial number of clusters $k=10$ ) to the image data resulting from the filtering process and a number of experimental results are depicted in Figs. 9 and 10. The experimental results 
depicted in Figs. 9 and 10 indicate that the proposed algorithm outperforms the VMF3, VMF5 and $\mathrm{IN}_{\mathrm{EST}}$ GVF-AM noise removal strategies when they are analyzed with respect to the efficiency of the smoothing process and preservation of contextual features.

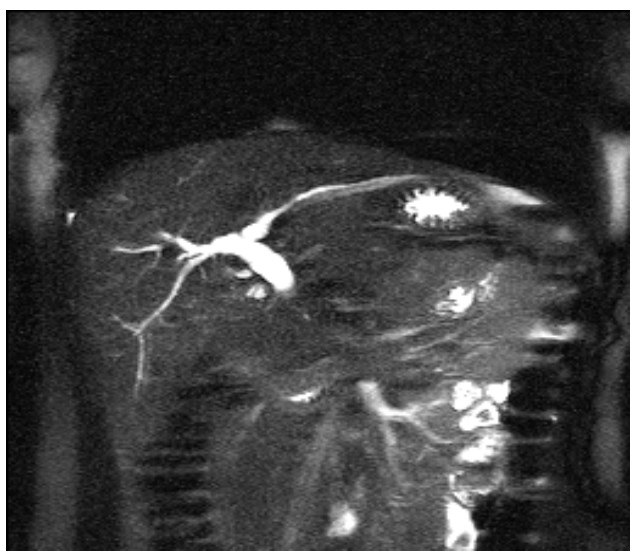

(a)

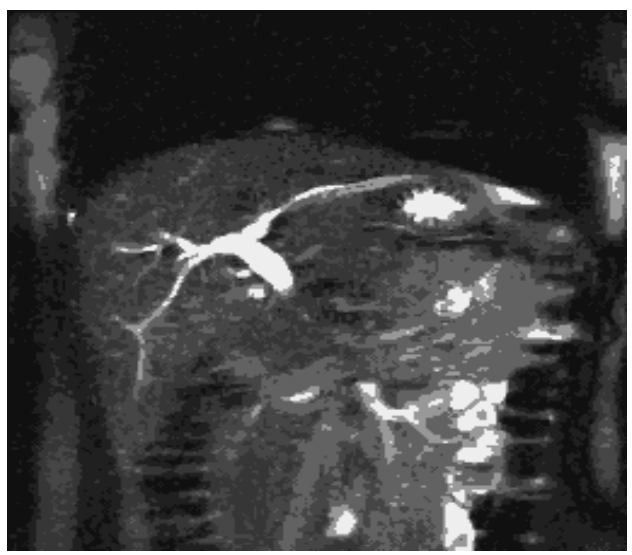

(b)

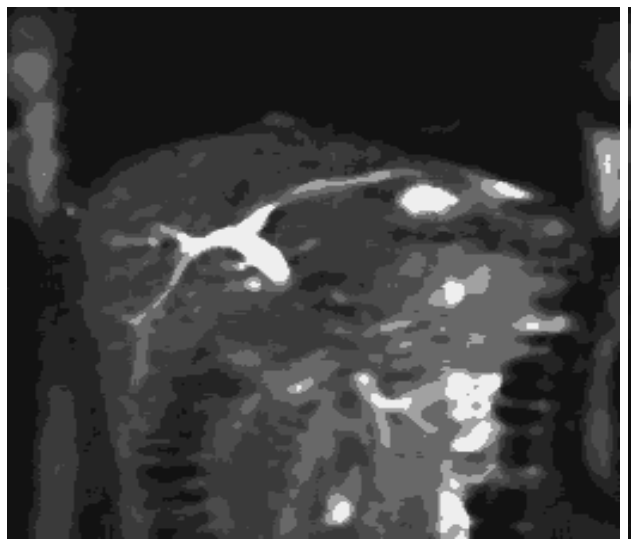

(c)

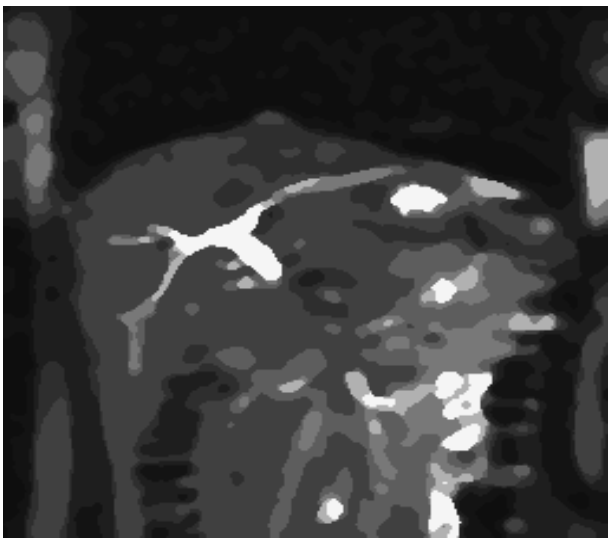

(d)

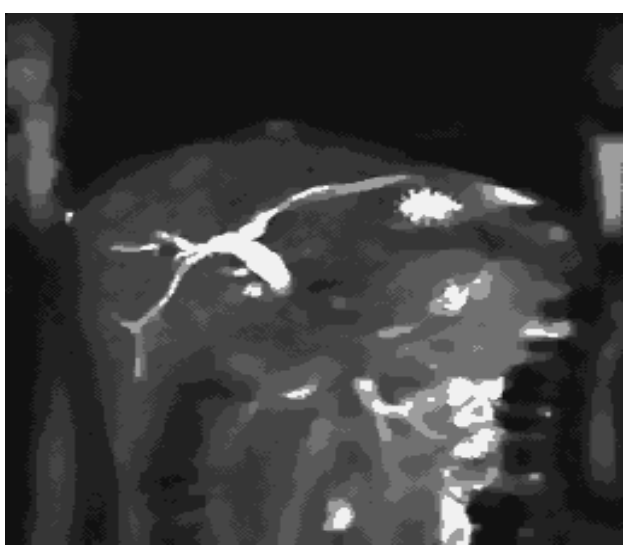

(e)

Fig. 9. Experimental results when the data smoothing algorithms have been applied to image segmentation tasks. (a) Original

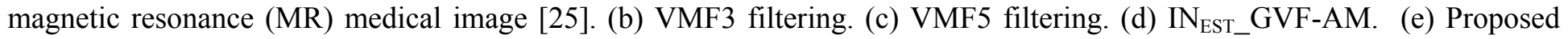
algorithm (MS-AD-VMF, VMF window $\Psi=5 \times 5$ ). Note the improved segmentation of the coherent regions that is achieved when the original image data shown in (a) has been filtered with the proposed MS-AD-VMF algorithm. 


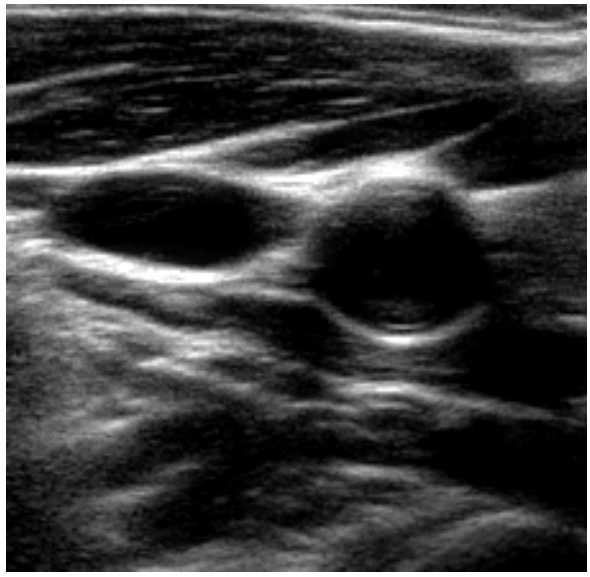

(a)

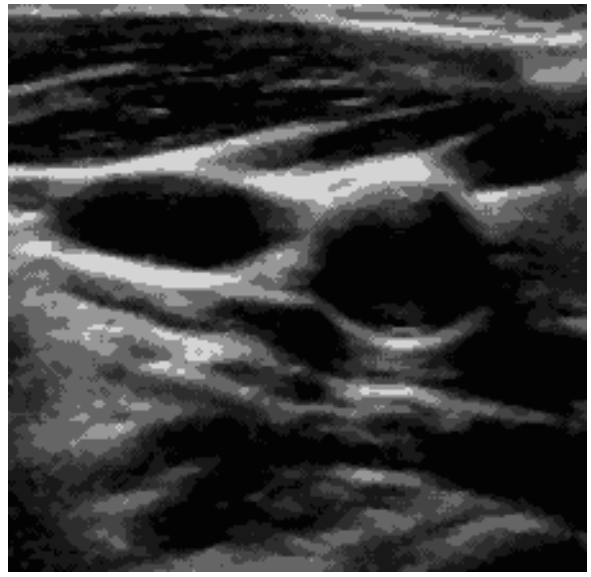

(b)

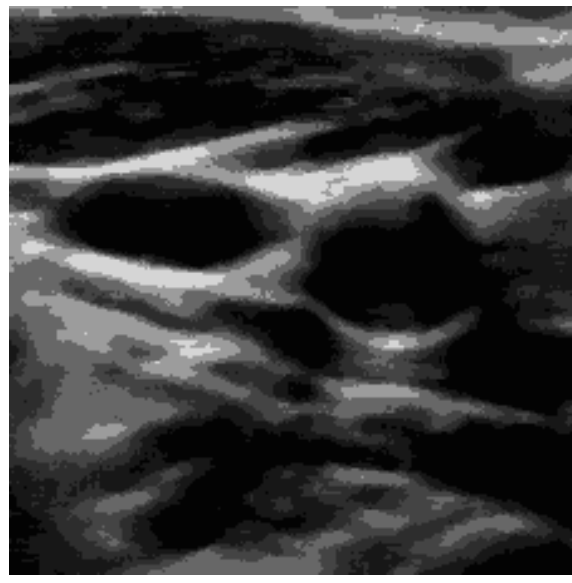

(c)

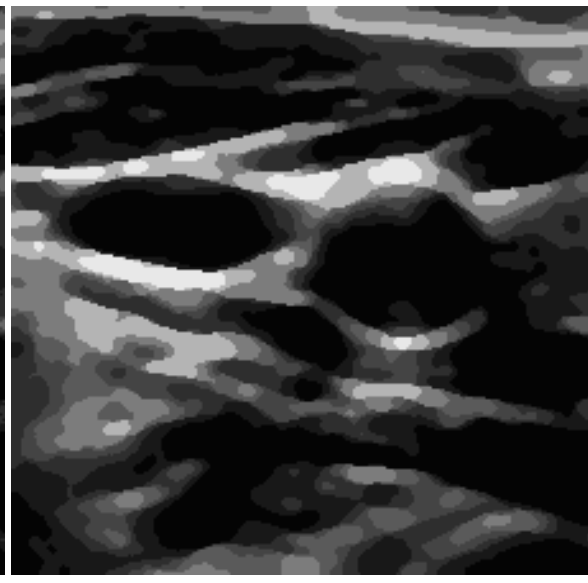

(d)

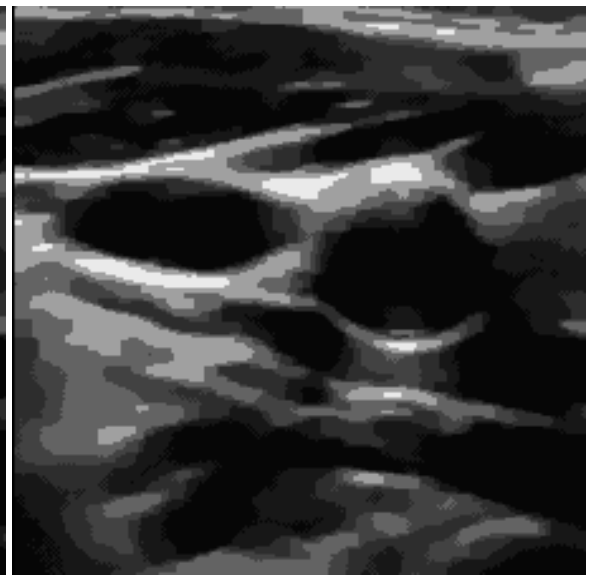

(e)

Fig. 10. Experimental results when the data smoothing algorithms have been applied to image segmentation tasks. (a) Original carotid ultrasound image [26]. (b) VMF3 filtering. (c) VMF5 filtering. (d)

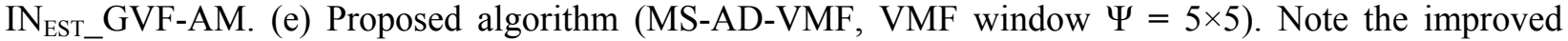
segmentation of the coherent regions that is achieved when the original image data shown in (a) has been filtered with the proposed MS-AD-VMF algorithm.

\section{Conclusions}

The aim of this paper was to detail the development of a multi-phase data smoothing scheme that is able to restore digital images corrupted by multimodal image noise. The proposed strategy is generic and it can be used in conjunction with any noise removal method that incorporates PDE models in order to achieve feature preservation. In this paper, we demonstrated that the inclusion of the vector median 
filtering (VMF) into the formulation of the anisotropic diffusion produced a more robust noise removal scheme that is able to locally adapt to the characteristic of the image noise. The proposed noise removal scheme was quantitatively evaluated using standard metrics such as PSNR, edge preservation index (Epi) and $\Delta \mathrm{E}_{00}$ index and its performance has also been assessed when applied to image segmentation tasks. Our future studies will focus on the development of more sophisticated statistical models that can be applied to detect the pixels that are corrupted by non-Gaussian distributed noise and additional work will be concerned with the optimisation of the proposed noise removal strategy when included in the implementation of image analysis algorithms that target the segmentation of low contrast medical data.

\section{Acknowledgments}

This work was financially supported by the Office of the Vice-President for Research (OVPR), Dublin City University, Ireland, with additional support provided by RINCE and the National Biophotonics and Imaging Platform Ireland (NBIPI - PRTLI Cycle 4).

\section{References}

[1] R.H. Chan, C. Ho, and M. Nikolova, "Salt-and-pepper noise removal by median-type noise detectors and detail-preserving regularization", IEEE Transactions on Image Processing, vol. 14, no. 10, pp. 1479-1485, 2005.

[2] M. Sonka, V. Hlavac, and R. Boyle, Image processing, analysis and machine vision. $2^{\text {nd }}$ edition, PWS Boston, 1998.

[3] P. Perona and J. Malik, "Scale-space and edge detection using anisotropic diffusion", IEEE Transactions on Pattern Analysis and Machine Intelligence, vol. 12, no. 7, pp. 629-639, 1990.

[4] M.J. Black, G. Sapiro, D.H. Marimont, and D. Hegger, "Robust anisotropic diffusion", IEEE Transactions on Image Processing, vol. 7, no. 3, pp. 421-432, 1998. 
[5] O. Ghita, K. Robinson, M. Lynch, and P.F. Whelan, "MRI diffusion-based filtering: A note on performance characterisation”, Computerized Medical Imaging and Graphics, vol. 29, no. 4, pp. 267277, 2005.

[6] S.H. Lee, M.G. Kang, and K.T. Park, “CCD noise filtering based on 3-dimensional nonlinear partial differential equation", IEEE Transactions on Consumer Electronics, vol. 44, no. 3, pp. 1086-1090, 1998.

[7] G. Gilboa, N. Sochen, and Y.Y. Zeevi, "Forward-and-backward diffusion processes for adaptive image enhancement and denoising”, IEEE Transactions on Image Processing, vol. 11, no. 7, pp. 689703, 2002.

[8] J. Weickert, Anisotropic diffusion in image processing. Teubner-Verlag, Stuttgart, Germany, 1998.

[9] D.E. Ilea and P.F. Whelan, "Adaptive pre-filtering techniques for color image analysis", Proc. of the International Machine Vision \& Image Processing Conference, IEEE Computer Society Press, pp. $150-157,2007$.

[10] D.E. Ilea and P.F. Whelan, "CTex-An adaptive unsupervised segmentation algorithm based on colortexture coherence", IEEE Transactions on Image Processing, vol. 17, no. 10, pp. 1926-1939, 2008.

[11] G. Shapiro and D. Ringach, "Anisotropic diffusion of multivalued images with applications to color filtering”, IEEE Transactions on Image Processing, vol. 5, no. 11, pp. 1582-1586, 1996.

[12] J. Astola, P. Haavisto, and Y. Nuevo, "Vector median filters", Proc. IEEE, vol. 79, pp. 678-689, 1990.

[13] M. Celebi and Y. Aslandogan, "Robust switching vector median filter for impulsive noise removal", Journal of Electronic Imaging, vol. 17, no. 4, 043006, 2008.

[14] R. Lukac, “Adaptive vector median filtering”, Pattern Recognition Letters, vol. 24, no. 12, pp. 18891899, 2003.

[15] R. Lukac, B. Smolka, and K.N. Plataniotis, "Sharpening vector median filters", Signal Processing, vol. 87, no. 9, pp. 2085-2099, 2007. 
[16] Y. Shen and K.E. Barner, "Fuzzy vector median-based surface smoothing", IEEE Transactions on Visualization and Computer Graphics, vol. 10, no. 3, pp. 252-265, 2004.

[17] A. Maalouf, P. Carre, B. Augereau, and C. Fernandez-Maloigne, "Cooperation of the partial differential equation methods and the wavelet transform for the segmentation of multivalued images", Signal Processing: Image Communication, vol. 23, no. 1, pp. 14-30, 2008.

[18] C. Feddern, J. Weickert, B. Burgeth, and M. Welk, "Curvature-driven PDE for matrix-valued images", International Journal of Computer Vision, vol. 69, no. 1, pp. 93-107, 2006.

[19] S. Di Zenzo, "A note on the gradient of a multi-image", Computer Vision, Graphics, and Image Processing, vol. 33, no. 1, pp. 116-125, 1986.

[20] The Berkeley Segmentation Dataset and Benchmark (BSDB), 2001. http://www.eecs.berkeley.edu/Research/Projects/CS/vision/grouping/segbench

[21] O. Ghita, D.E. Ilea, and P.F. Whelan, "Image feature enhancement based on the time-controlled total variation flow formulation", Pattern Recognition Letters, vol. 30, no. 3, pp. 314-320, 2009.

[22] C. Tomasi and R. Manduchi, "Bilateral filtering for gray and color images", Proc of the $6^{\text {th }}$ International Conference on Computer Vision (ICCV), pp. 839-846, 1998.

[23] A. Buades, B. Coll, and J.M. Morel, "A review of image denoising algorithms, with a new one", Multiscale Modeling and Simulation, vol. 4, no. 2, pp. 490-530, 2005.

[24] D. Comaniciu and P. Meer, "Mean shift: A robust approach toward feature space analysis", IEEE Transactions on Pattern Analysis and Machine Intelligence, vol. 24, no. 5, pp. 603-619, 2002.

[25] Department of Radiology, Mater Misercordiae Hospital, Dublin 7, Ireland.

[26] Molecular and Cellular Therapeutics, Royal College of Surgeons in Ireland, Dublin 2, Ireland.

[27] F. Sattar, L. Floreby, G. Salomonsson, and B. Lovstrom, "Image enhancement based on a nonlinear multiscale method", IEEE Transactions on Image Processing, vol. 6, no. 6, pp. 888-895, 1997.

[28] O. Ghita and P.F. Whelan, "A new GVF-based image enhancement formulation for use in the presence of mixed noise”, Pattern Recognition, vol. 43, no. 8, pp. 2646-2658, 2010. 
[29] Z. Yang and M.D. Fox, "Speckle reduction and structure enhancement by multichannel median boosted anisotropic diffusion", EURASIP Journal on Signal Processing, vol.16, pp. 2492-2502, 2004.

[30] H. Yu and C.S. Chua, "GVF-based anisotropic diffusion models", IEEE Transactions on Image Processing, vol. 15, no. 6, pp. 1517-1524, 2006.

[31] M.R. Luo, G. Cui, and B. Rigg, "The development of the CIE 2000 colour-difference formula: CIEDE2000", Color Research and Applications, vol. 26, no. 5, pp. 340-350, 2001.

[32] G. Sharma, W. Wu, and E.N. Dalal, "The CIEDE2000 color-difference formula: Implementation notes, supplementary test data, and mathematical observations", Color Research and Applications, vol. 30 , no. 1 , pp. 21-30, 2005. 